\title{
Mapping anhedonia-specific dysfunction in a transdiagnostic approach: an ALE meta-analysis
}

\author{
Bei Zhang ${ }^{1} \cdot$ Pan Lin ${ }^{2} \cdot$ Huqing Shi ${ }^{3}$ - Dost Öngür ${ }^{4} \cdot$ Randy P. Auerbach ${ }^{4}$. \\ Xiaosheng Wang ${ }^{5} \cdot$ Shuqiao Yao ${ }^{1} \cdot$ Xiang Wang $^{1}$
}

Published online: 20 October 2015

(C) The Author(s) 2015. This article is published with open access at Springerlink.com

\begin{abstract}
Anhedonia is a prominent symptom in neuropsychiatric disorders, most markedly in major depressive disorder (MDD) and schizophrenia (SZ). Emerging evidence indicates an overlap in the neural substrates of anhedonia between MDD and SZ, which supported a transdiagnostic approach. Therefore, we used activation likelihood estimation (ALE) meta-analysis of functional magnetic resonance imaging studies in MDD and SZ to examine the neural bases of three subdomains of anhedonia: consummatory anhedonia, anticipatory anhedonia and emotional processing. ALE analysis focused specifically on MDD or SZ was used later to dissociate specific anhedonia-related neurobiological impairments from potential disease general impairments. ALE results
\end{abstract}

Electronic supplementary material The online version of this article (doi:10.1007/s11682-015-9457-6) contains supplementary material, which is available to authorized users.

Xiang Wang

wang0916xia@gmail.com

1 Medical Psychological Institute, The Second Xiangya Hospital of Central South University, 139 Renmin (M) Road,

Changsha, Hunan 410011, People's Republic of China

2 Key Laboratory of Biomedical Information Engineering of Education Ministry, Institute of Biomedical Engineering, Xi'an Jiaotong University, Xi'an, Shanxi 710049, People's Republic of China

3 Department of Psychology, Shanghai Normal University, Shanghai 200234, People's Republic of China

4 Harvard Medical School and McLean Hospital, 115 Mill Street, Belmont, MA 02478, USA

5 Department of Anatomy and Neurobiology, Xiangya School of Medicine, Central South University, Changsha 410013, People's Republic of China revealed that consummatory anhedonia was associated with decreased activation in ventral basal ganglia areas, while anticipatory anhedonia was associated with more substrates in frontal-striatal networks except the ventral striatum, which included the dorsal anterior cingulate, middle frontal gyrus and medial frontal gyrus. MDD and SZ patients showed similar neurobiological impairments in anticipatory and consummatory anhedonia, but differences in the emotional experience task, which may also involve affective/mood general processing. These results support that anhedonia is characterized by alterations in reward processing and relies on frontal-striatal brain circuitry. The transdiagnostic approach is a promising way to reveal the overall neurobiological framework that contributes to anhedonia and could help to improve targeted treatment strategies.

Keywords Anhedonia - Activation likelihood estimation (ALE) - Meta-analysis · Transdiagnostic $\cdot$ Major depressive disorder $\cdot$ Schizophrenia

\section{Introduction}

Anhedonia is defined as 'markedly diminished interest or pleasure in all, or almost all, activities most of the day, nearly every day' (Association and Association 1994). Although anhedonia has long been considered a prominent symptom in neuropsychiatric disorders, especially major depressive disorder (MDD) and schizophrenia (SZ) (Association and Association 1994), its underlying neurobiological mechanisms remain poorly understood. Recently, evidence has emerged to indicate overlap of behavioral, cognitive processing and neurobiological abnormalities between MDD and SZ patients with marked clinical anhedonia (Gradin et al. 2011; Whitton et al. 2015). As a result of this finding and the advocacy of 
the NIMH Research Domain Criteria (RDoC) project (Insel et al. 2010), investigators are seeking to use the basic behavioral dimension of functioning, rather than traditional diagnostic categories, to identify transdiagnostic neural markers of anhedonia (CorralFrias et al. 2015; Cuthbert and Insel 2013; Markou et al. 2009). A quantitative meta-analysis of pooled neuroimaging studies of anhedonia in MDD and SZ, discussed within the RDoC framework, is a promising approach to investigate the neural substrates of anhedonia. However, it is important to be mindful of the complexity and multifaceted nature of clinical anhedonia to understand neuroimaging studies in this field.

Based on the latest studies, anhedonia is a multidimensional construct and should not simply be considered as 'loss of an ability to experience pleasure'. Deficits in other reward processes, such as valuation, motivation and decision-making, may lead to behaviors that can be interpreted as anhedonia (Der-Avakian and Markou 2012; Gold et al. 2008; Leventhal et al. 2006). Hence, distinguishing the deficits in different cognitive subcomponents of anhedonia is essential to identify its neurobiological substrates. Some investigators have suggested dichotomizing anhedonia into 'consummatory anhedonia' (the hedonic response to rewards) and 'anticipatory anhedonia' (diminished motivation to pursue rewards) (Treadway and Zald 2011). Others hold that studies should move away from conceptualization of anhedonia as a steady-state, mood-like phenomena, and instead focus on the reward-related motivational/decisionalmaking aspect (Treadway et al. 2012; Whitton et al. 2015). Still others propose to bridge the gap between preclinical and clinical studies by isolating the neural substrates of various processes, such as sensing a pleasant stimulus or anticipating expected rewards, computing value and associated costs, determining effort required, deciding to obtain the reward, increasing motivation and performing the action (Der-Avakian and Markou 2012). Changing research strategies and models demand more complicated and elaborate experimental designs to investigate the underlying neural bases of anhedonia.

Several experimental paradigms have been developed in last decade to explore the specific processes of anhedonia beyond hedonic capacity, such as anticipation, motivation and reinforcement learning. However, only a few task-based functional magnetic resonance imaging (fMRI) studies have separated anhedonia deficits into different processing stages. Early neuroimaging studies frequently adopted the passive picture-viewing task based on the viewpoint that anhedonia represents a diminished responsiveness to positive-valence stimuli (Hooker et al. 2014; Mitterschiffthaler et al. 2003; Shi et al. 2013). More recently, some experimental reward paradigms, especially the monetary incentive delay task (MID) (Admon et al. 2015; Balodis and Potenza 2015; Elman et al. 2009), have been increasingly used to detect neural substrates of anhedonia. Those studies have typically explored brain activity impairments in experience/ consummatory and motivational/anticipatory stages separately, and they discussed the link between anhedonia and the reward-processing components of 'liking' and 'wanting' from the preclinical literature (Berridge and Robinson 2003; Dillon et al. 2008). Specifically, the consummatory stage was primarily associated with dysfunctions of reward coding and evaluation, and the anticipatory stage was associated with advanced cognitive functions, such as motivation and decision-making. Recently, although other paradigms have been developed to assess a specific aspect of anhedonia-related processing, such as the Effort Expenditure for Rewards Task (EEfRT), which focuses on motivation and effort-based decision-making (Fervaha et al. 2013; Treadway et al. 2009; Yang et al. 2014) and the Probabilistic Learning Task (PRT), which focuses on reward learning (Pizzagalli et al. 2008), no neuroimaging studies have used these two paradigms in MDD or SZ patients. In fact, to date the majority of studies have explored the neurobiology of the three subdomains of anhedonia, which involve different neurocognitive process and functional neuroanatomy bases: consummatory anhedonia, anticipatory anhedonia and emotional (positive stimuli) processing (Berridge and Robinson 2003; Dillon et al. 2008; Smoski et al. 2011).

Neuroimaging studies of the three subdomains of anhedonia in MDD and SZ patients have reached inconsistent and even contradictory findings. For instance, several studies that used a reward-related task, most often MID, indicated ventral striatal blunting in depressed adults (Knutson et al. 2008; Kumar et al. 2008; Ubl et al. 2015) and even in never-depressed youth with family history of MDD (Gotlib et al. 2010). Another study using the same experimental paradigm (MID) showed no significant group difference in ventral striatum between unmedicated MDD and controls after correction (Pizzagalli et al. 2009). Furthermore, Pizzagalli et al. (2008) reported decreased activation in dorsal anterior cingulate (dACC) during the MID task in MDD patients compared with healthy controls, whereas other studies found increased activation of dACC in MDD (Gorka et al. 2014; Knutson et al. 2008). Similar contradictory results have been observed in SZ patients, especially in the experience/consummatory stage. When presented with reward stimuli, SZ patients showed impairments in both ventral striatal and prefrontal areas (da Silva Alves et al. 2013; Walter et al. 2009a), impairments in prefrontal cortex but not basal ganglia areas (Waltz et al. 2010) or no impairments in either area (Mucci et al. 2015; Simon et al. 2010). These 
inconsistencies among studies may reflect differences in sample size, sample characteristics (average age, sexual ratio, medicated or not), paradigms used, and fMRI analysis methods.

The development of meta-analyses methods for neuroimaging data provide a valuable tool for combining data across diverse studies and building consensus in identification of neuroanatomical correlates of specific behavior. To date, the main methods used for the metaanalyses of neuroimaging data could be divided into two categories: the regions of interest (ROI) based metaanalytic methods, and the voxel-based meta-analytic methods. The ROI-based methods allow for optimal statistical analyses but are based on a priori hypotheses therefore being affected by a limited and potentially biased inclusion of brain regions (Rotge et al. 2009; Radua and Mataix-Cols 2012). On the other hand, voxel-based methods have a more exhaustive and unbiased inclusion of studies but have some statistically limitation. Activation likelihood estimation (ALE), proposed by Turkeltaub and Laird (Turkeltaub et al. 2002; Laird et al. 2005), is probably the most common algorithm for voxel-based meta-analyses.

Besides the clear mathematical logic and operation steps, ALE method has several advantages over ROIbased meta-analyses through inputting the foci of activation instead of labels, weighting the foci by the number of participants in each study, yielding a quantitative estimate of the probability of activation, identifying common activations across different studies (Laird et al. 2005; Wager et al. 2007; Eickhoff et al. 2009; Eickhoff et al. 2012). More importantly, the recent change from fixed-effects to random-effects inference in analyses (Eickhoff et al. 2009) and the revision for multiple comparison correction (Eickhoff et al. 2012) made ALE to become a more reliable statistic meta-analysis approach.

Therefore, the ALE meta-analysis method was used in this review to make an objective, systematic, and quantitative analysis of the previous literature and synthesize the anhedonia-related, task-based fMRI findings in MDD and SZ from three subdomains of anhedonia: consummatory anhedonia, anticipatory anhedonia, and emotional processing. Specifically, we first performed the within-group ALE metaanalyses to provide a context for interpreting the anhedoniarelated between-group differences. In the between-group analysis, the transdiagnostic ALE analysis of the literature on MDD and SZ was examined to provide an integrated framework of the neural bases of anhedonia, and the analysis focused specifically on MDD or SZ was used later to dissociate specific anhedonia-related neurobiological impairments from potential disease general impairments. The transdiagnostic approach provides the opportunity to identify an overall neurobiological framework for a specific symptom or behavior across multiple disease states and has the potential to improve targeted treatment strategies.

\section{Methods}

\section{Data sources}

Studies focused on the neurobiology of anhedonia in patients with MDD or SZ were identified through computerized literature searches using PubMed and Web of Science. We reviewed all papers published in the English language up to August 1, 2015. The key words used for the search were 'anhedonia', 'hedonic', 'emotional withdrawal', 'pleasure deficit', 'apathetical social withdrawal', 'functional magnetic resonance' and 'fMRI'. A total of 571 English publications were initially identified using this process. Another three articles were obtained from reference lists of prior reviews (Whitton et al. 2015; Zhang et al. 2013). In summary, these search procedures yielded an initial pool of 574 potential articles for inclusion.

In prior neuroimaging studies focused on anhedonia, the most common paradigms adopted were the MID, reinforcement learning task (RLT) and emotional experience task (EET). For MID, RLT or some modified reward tasks, subjects initially see a cue indicating that they will have an opportunity to obtain a certain amount of reward (e.g., money), then they perform a task, and they receive immediate feedback (by obtaining or not obtaining the reward). The process between the cue and the task refers to anticipation stage, and the in-the-moment experience during receipt of a reward refers to consummatory stage. Besides, the emotional experience task (EET) is associated with a passive viewing of positive-valence stimuli that requires no response. Therefore, based on different cognitive process, we divided anhedonia into three subdomains: anticipatory anhedonia, consummatory anhedonia, and emotional processing.

\section{Inclusion criteria}

For the current meta-analysis, once duplicate articles were removed, the following eight inclusion criteria were utilized: (1) first-hand empirical studies; (2) subjects were healthy controls or patients with either MDD or SZ; (3) MDD or SZ were diagnosed according to DSM-IV(-TR) or ICD-10; (4) studies focused on anhedonia though taskrelated paradigms; (5) studies examined neuronal activity related to anhedonia using fMRI; (6) studies identified foci of task-related neural changes in active conditions (e.g., emotional picture or monetary gain) and/or contrasted an active and a control condition (neutral 
picture or fixation cross); (7) coordinates were reported in either standard Talairach space or Montreal Neurologic Institute (MNI) space. Figure 1 shows the process of study identification and selection. From the identification and selection of studies, coordinate results of withingroup activations and between-group differences were divided into 4 groups: activations in patients, activations in healthy controls, increases in patients relative to controls, and increases in controls relative to patients.

\section{Activation likelihood estimation (ALE) procedure}

Meta-analysis was performed using the ALE software implemented in GingerALE version 2.3.2 (http://brainmap.org). This
Fig. 1 Flowchart describing the process of study identification and selection

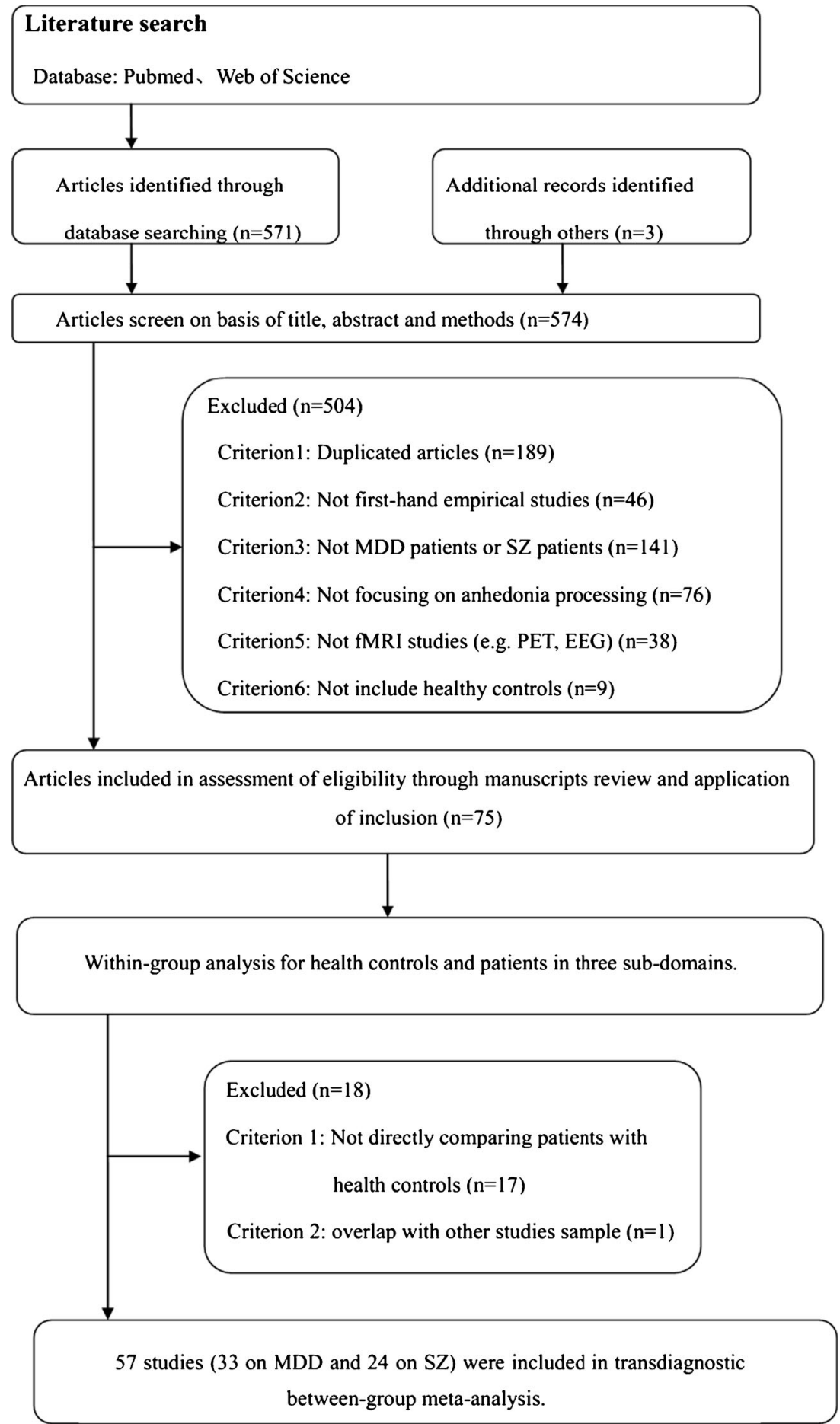


ALE version used a random effect model and weighted for sample size of the original studies (Laird et al. 2005). In ALE, activation foci reported in original studies are treated as 3D Gaussian distributions centered at the reported coordinates. Activation probabilities are then calculated for each standardspace voxel to construct ALE maps for contrasts of interest. To determine the reliability of the ALE map, null-distributions are generated by analyzing the distribution of ALE values across independent studies, which is conceptually similar to using permutation tests of individual voxels across experiments. The observed values in the ALE distribution are then compared to the null distribution in order to assign probability estimates to the observed (experimental) data.

For the present meta-analysis, single studies were used respectively to perform meta-analyses of anhedonia for each of the three subdomains. Coordinates of the foci of activation reported in MNI were transformed to Talairach space using the icbm2tal in GingerALE. The threshold of statistical significance was set at $p<0.01$ and corrected for multiple testing using the false discovery rate (FDR) with a minimum cluster size of $400 \mathrm{~mm}^{3}$. For visualization, whole-brain maps of thresholded ALE maps were imported into multi-image analysis GUI (MANGO; http://ric.uthscsa.edu/mango) and overlaid onto a standardized anatomical template in Talairach space (www.brainmap.org/ale/colin1.1.nii).

\section{Results}

\section{Article inclusion}

Figure 1 shows the study selection flowchart, and Table 1 shows the pooled data for all contrasts. A total of 32 articles with 684 healthy controls reporting 353 foci, and 28 articles with 562 patients reporting 214 foci were included in withingroup analysis. A total of 57 articles (33 articles on MDD and 24 on SZ) with 986 patients and 1041 healthy controls and reporting 453 foci were included in the transdiagnostic metaanalysis. There were no significant differences in age or sex between patients $(34.33 \pm 8.55$ years old; $51.09 \%$ men) and healthy controls ( $32.12 \pm 7.09$ years old; $51.71 \%$ men), MDD patients ( $36.40 \pm 8.79$ years old; $40.82 \%$ men) and healthy controls (33.85 \pm 8.42 years old; $39.80 \%$ men) or between SZ patients ( $30.75 \pm 6.96$ years old; $63.64 \%$ men) and healthy controls ( $31.85 \pm 3.70$ years old; $71.33 \%$ men).

\section{Within-group ALE analysis}

\section{Healthy controls}

For reward consummatory (16 studies and 160 foci), ALE revealed a set of subcortical areas, including bilateral globus pallidus (GPe), bilateral red nucleus, right caudate body, left substania nigra (SN) and right putamen, left parahippocampal gyrus, as well as left medial frontal gyrus (mPFC). For reward anticipation (17 studies and 99 foci), ALE results showed robust activation of a broad corticalsubcortical network, including left GPe, right caudate head, left caudate body, bilateral red nucleus, right inferior frontal cortex (IFG), right superior temporal gyrus (STG), right insula, left mPFC and some midbrain areas, such as mammillary body. For the emotional processing ( 9 studies and 94 foci), several regions in frontal gyrus were activated to a greater degree, such as mPFC, MFG and IFG, as well as the middle temporal gyrus and fusiform gyrus $(p<0.01$, FDR corrected, cluster size $>400 \mathrm{~mm} 3$, Table 2 and Fig. 2).

\section{Patients (mixed MDD and SZ)}

For reward consummatory (11 studies and 68 foci), ALE analysis found seven statistically significant activation clusters, including left $\mathrm{GPe}$, right caudate head, left red nucleus, right insula, left anterior cingulate gyrus (ACC), IFG and amydala. Reward anticipation (14 studies and 63 foci) was related to the significant activation of putamen, superior frontal gyrus (SFG) and IFG. As to the emotional processing (7 studies and 83 foci), increased likelihood of activation was observed in several frontal gyrus, temporal gyrus, occipital gyrus, as well as caudate body $(p<0.01$, FDR corrected, cluster size $>400 \mathrm{~mm} 3$, Table 2 and Fig. 2). The results of within-group analysis results in MDD or SZ separately showed the similarly distribution and activation pattern (details see Table S1 and Fig. S1).

\section{Between-group ALE analysis in a transdiagnostic approach across MDD and SZ}

\section{Consummatory anhedonia}

For consummatory anhedonia (29 studies and 151 foci), ALE analysis revealed five statistically significant clusters with decreased likelihood of activation in patients compared to controls (Fig. 3 and Table 3), including bilateral caudate head and body, bilateral GPe, left putamen, right red nucleus, left ventral lateral nucleus and pulvinar, as well as MTG $(p<0.01$, FDR corrected, cluster size $>400 \mathrm{~mm}^{3}$ ). No brain regions showed increased likelihood of activation in patients compared to controls.

\section{Anticipatory anhedonia}

For anticipatory anhedonia (30 studies and 119 foci), prefrontal cortex and striatal areas showed significantly different activity (Fig. 3 and Table 3). Decreased likelihood of activation was observed in bilateral caudate head, left 


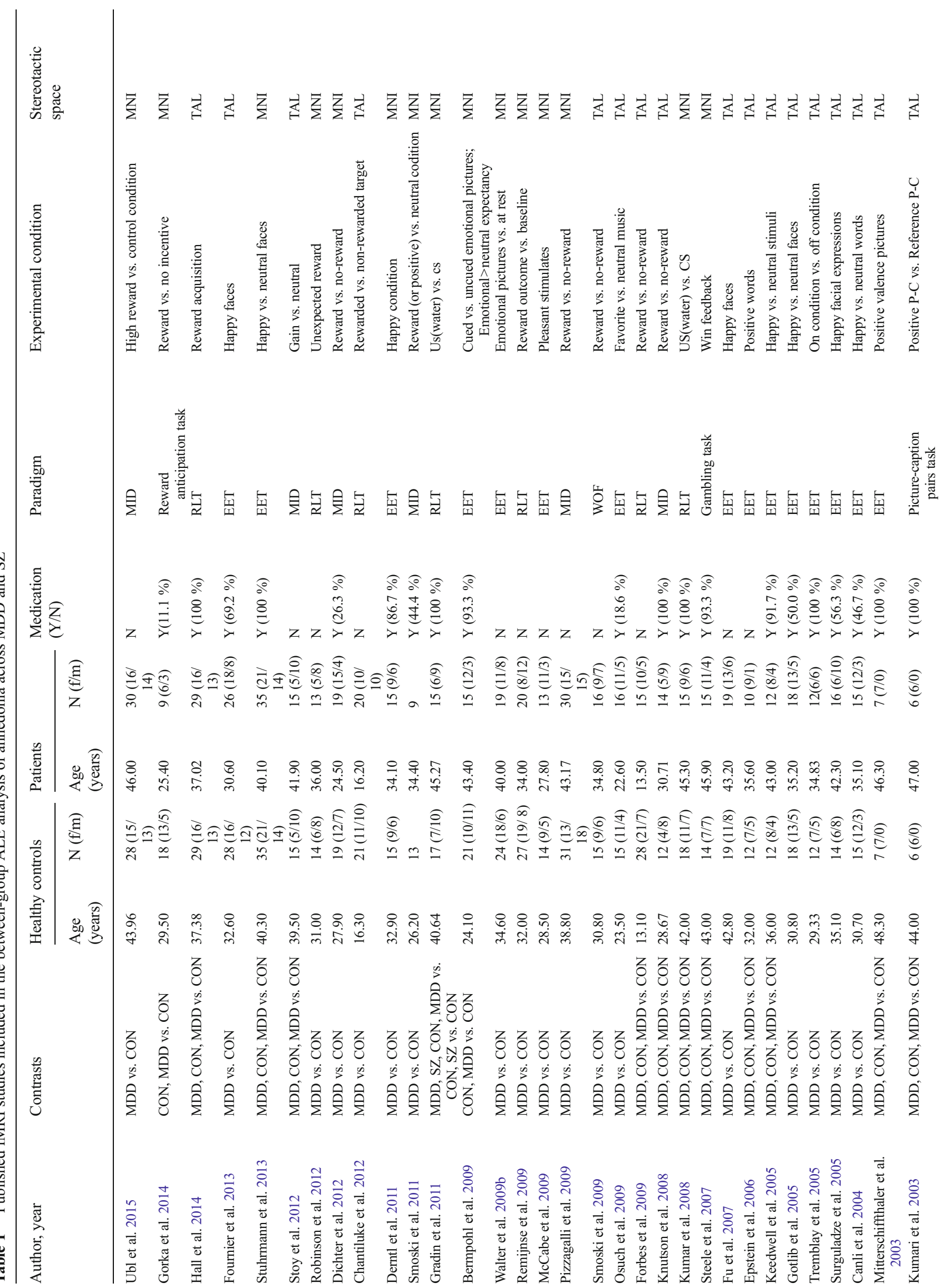




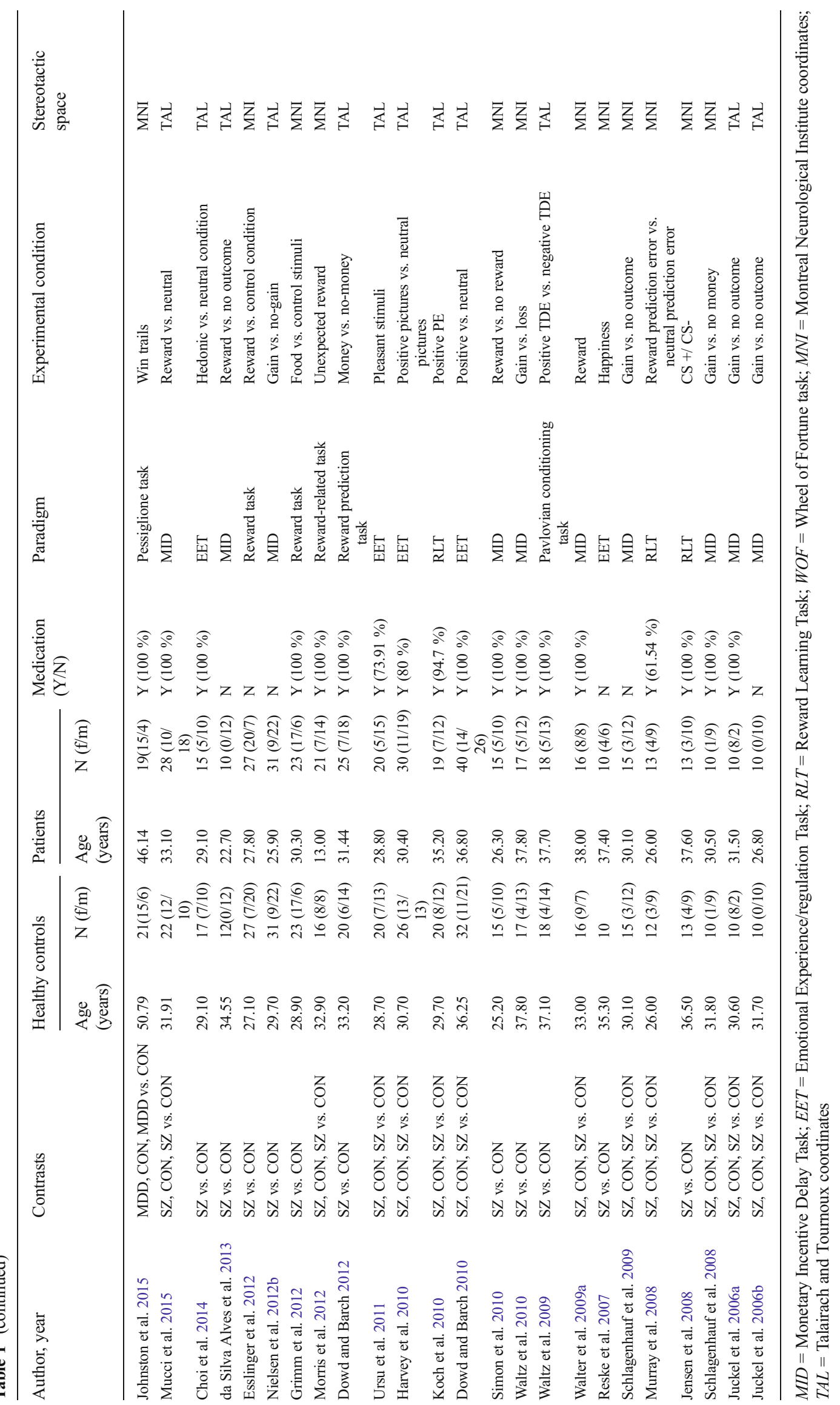


Table 2 Within-group ALE meta-analysis results in healthy controls and patients (mixed MDD and SZ)

\begin{tabular}{|c|c|c|c|c|c|c|c|}
\hline Cluster & Anatomical region & BA & $\mathrm{X}$ & $\mathrm{Y}$ & Z & Volume $\mathrm{mm}^{3}$ & Maximum ALE Value $\left(10^{-3}\right)$ \\
\hline \multicolumn{8}{|c|}{ Healthy controls } \\
\hline \multicolumn{8}{|c|}{ Reward consummatory } \\
\hline \multirow[t]{4}{*}{1} & R Lateral Globus Pallidus & & 10 & 6 & -2 & 10384 & 25.07 \\
\hline & L Lateral Globus Pallidus & & -10 & 4 & 0 & & 24.63 \\
\hline & L Parahippocampal Gyrus & 34 & -18 & 0 & -12 & & 13.16 \\
\hline & R Caudate Body & & 8 & 18 & 6 & & 12.73 \\
\hline 2 & L Medial Frontal Gyrus. & & -4 & 48 & 2 & 2864 & 21.02 \\
\hline \multirow[t]{3}{*}{3} & R Red Nucleus & & 4 & -22 & -8 & 1432 & 14.40 \\
\hline & L Red Nucleus & & -4 & -16 & -4 & & 9.87 \\
\hline & L Substania Nigra & & -8 & -16 & -12 & & 8.77 \\
\hline 4 & R Putamen & & 26 & 0 & -6 & 440 & 10.84 \\
\hline \multicolumn{8}{|c|}{ Reward anticipation } \\
\hline \multirow[t]{9}{*}{1} & L Lateral Globus Pallidus & & -12 & 6 & 0 & 20736 & 49.99 \\
\hline & R Caudate Head & & 10 & 6 & 0 & & 46.31 \\
\hline & R Red Nucleus & & 6 & -26 & -8 & & 19.19 \\
\hline & L Red Nucleus & & -6 & -22 & -8 & & 14.62 \\
\hline & L Caudate Body & & -12 & 4 & 18 & & 11.84 \\
\hline & R Subthalamic Nucleus & & 12 & -12 & -8 & & 10.90 \\
\hline & L Mammillary Body & & 0 & -12 & -10 & & 10.89 \\
\hline & R Mammillary Body & & 4 & -12 & -8 & & 10.89 \\
\hline & R Hypothalamus & & 10 & -6 & -8 & & 10.87 \\
\hline \multirow[t]{2}{*}{2} & R Inferior Frontal Gyrus & 47 & 50 & 14 & -2 & 712 & 12.80 \\
\hline & R Superior Temporal Gyrus & 38 & 52 & 18 & -8 & & 10.66 \\
\hline 3 & R Insula & 13 & 36 & 16 & 6 & 552 & 11.67 \\
\hline 4 & L Medial Frontal Gyrus & 10 & -2 & 54 & 4 & 424 & 10.84 \\
\hline 5 & L Anterior Lobe.Culmen. & & 0 & -60 & -6 & 416 & 10.58 \\
\hline \multicolumn{8}{|c|}{ Emotional processing } \\
\hline 1 & L Medial Frontal Gyrus & 32 & -8 & 12 & 46 & 1000 & 18.60 \\
\hline 2 & R Posterior Lobe.Declive & & 34 & -52 & -12 & 768 & 20.80 \\
\hline 3 & L Medial Frontal Gyrus & 9 & 0 & 46 & 32 & 688 & 16.68 \\
\hline 4 & L Inferior Frontal Gyrus & & -42 & 18 & 2 & 624 & 16.42 \\
\hline 5 & R Middle Temporal Gyrus & 39 & 46 & -72 & 14 & 528 & 16.92 \\
\hline 6 & R Fusiform Gyrus & 19 & 24 & -80 & -10 & 560 & 16.04 \\
\hline 7 & R Inferior Frontal Gyrus & 9 & 42 & 8 & 30 & 520 & 17.43 \\
\hline \multirow[t]{2}{*}{8} & L Medial Frontal Gyrus & 10 & -2 & 60 & -2 & 472 & 12.04 \\
\hline & L Medial Frontal Gyrus & 10 & -2 & 50 & -4 & & 10.96 \\
\hline 9 & L Posterior Lobe.Declive. & & -30 & -56 & -14 & 456 & 17.93 \\
\hline 10 & R Cuneus & 19 & 28 & -84 & 32 & 496 & 17.23 \\
\hline 11 & R Anterior Cingulate & 32 & 6 & 40 & 0 & 456 & 17.93 \\
\hline \multicolumn{8}{|c|}{ Patients (mixed MDD and SZ) } \\
\hline \multicolumn{8}{|c|}{ Reward consummatory } \\
\hline \multirow[t]{3}{*}{1} & L Lateral Globus Pallidus & & -12 & 4 & 0 & 2456 & 13.93 \\
\hline & L Parahippocampal Gyrus & 34 & -24 & 0 & -10 & & 13.69 \\
\hline & L Putamen & & -14 & 8 & -8 & & 12.20 \\
\hline \multirow[t]{2}{*}{2} & L Anterior Cingulate & 10 & -4 & 54 & -2 & 1952 & 18.72 \\
\hline & L Anterior Cingulate & 24 & -6 & 38 & 2 & & 9.12 \\
\hline 3 & $\mathrm{R}$ Insula & 13 & 38 & 4 & -4 & 1544 & 14.48 \\
\hline 4 & R Amydala & & 22 & -2 & -10 & 1424 & 16.94 \\
\hline 5 & R Caudate Head & & 6 & 8 & 0 & 1192 & 16.25 \\
\hline
\end{tabular}


Table 2 (continued)

\begin{tabular}{|c|c|c|c|c|c|c|c|}
\hline Cluster & Anatomical region & BA & $\mathrm{X}$ & $\mathrm{Y}$ & Z & Volume $\mathrm{mm}^{3}$ & Maximum ALE Value $\left(10^{-3}\right)$ \\
\hline 6 & L Red Nucleus & & 0 & -22 & -8 & 1144 & 11.76 \\
\hline 7 & L Inferior Frontal Gyrus & 9 & -54 & 12 & 30 & 488 & 12.78 \\
\hline \multicolumn{8}{|c|}{ Reward anticipation } \\
\hline 1 & R Putamen & & 14 & 8 & -2 & 3168 & 29.48 \\
\hline 2 & L Putamen & & -14 & 10 & -4 & 2536 & 16.57 \\
\hline 3 & R Inferior Frontal Gyrus & 9 & 54 & 20 & 26 & 528 & 13.44 \\
\hline 4 & L Superior Frontal Gyrus & 9 & -1 & 49 & 32 & 400 & 10.66 \\
\hline \multicolumn{8}{|c|}{ Emotional processing } \\
\hline 1 & R Declive & & 34 & -52 & -12 & 1168 & 21.69 \\
\hline 2 & R Middle Temporal Gyrus & 39 & 46 & -72 & 14 & 816 & 17.43 \\
\hline 3 & R Inferior Frontal Gyrus & 9 & 44 & 8 & 30 & 776 & 18.06 \\
\hline 4 & L Declive & & -30 & -56 & -14 & 680 & 18.96 \\
\hline 5 & $\mathrm{R}$ Cuneus & 19 & 29 & -85 & 32 & 576 & 17.52 \\
\hline 6 & L Caudate Body & & -18 & 20 & 14 & 496 & 11.39 \\
\hline 7 & L Middle Occipital Gyrus & 19 & -44 & -78 & 2 & 432 & 11.38 \\
\hline 8 & L Superior Frontal Gyrus & 9 & -6 & 52 & 24 & 432 & 11.89 \\
\hline
\end{tabular}

$p<0.01$, FDR corrected, cluster size $>400 \mathrm{~mm}^{3} ; B A=$ Brodmann area

Fig. 2 Significant ALE maps (FDR corrected, $p<0.01$, cluster size $>400 \mathrm{~mm} 3$ ) of within-group analysis in healthy control group (HC) and transdiagnostic patient group (mixed MDD and SZ) for reward consummatory, reward anticipation, and emotional processing

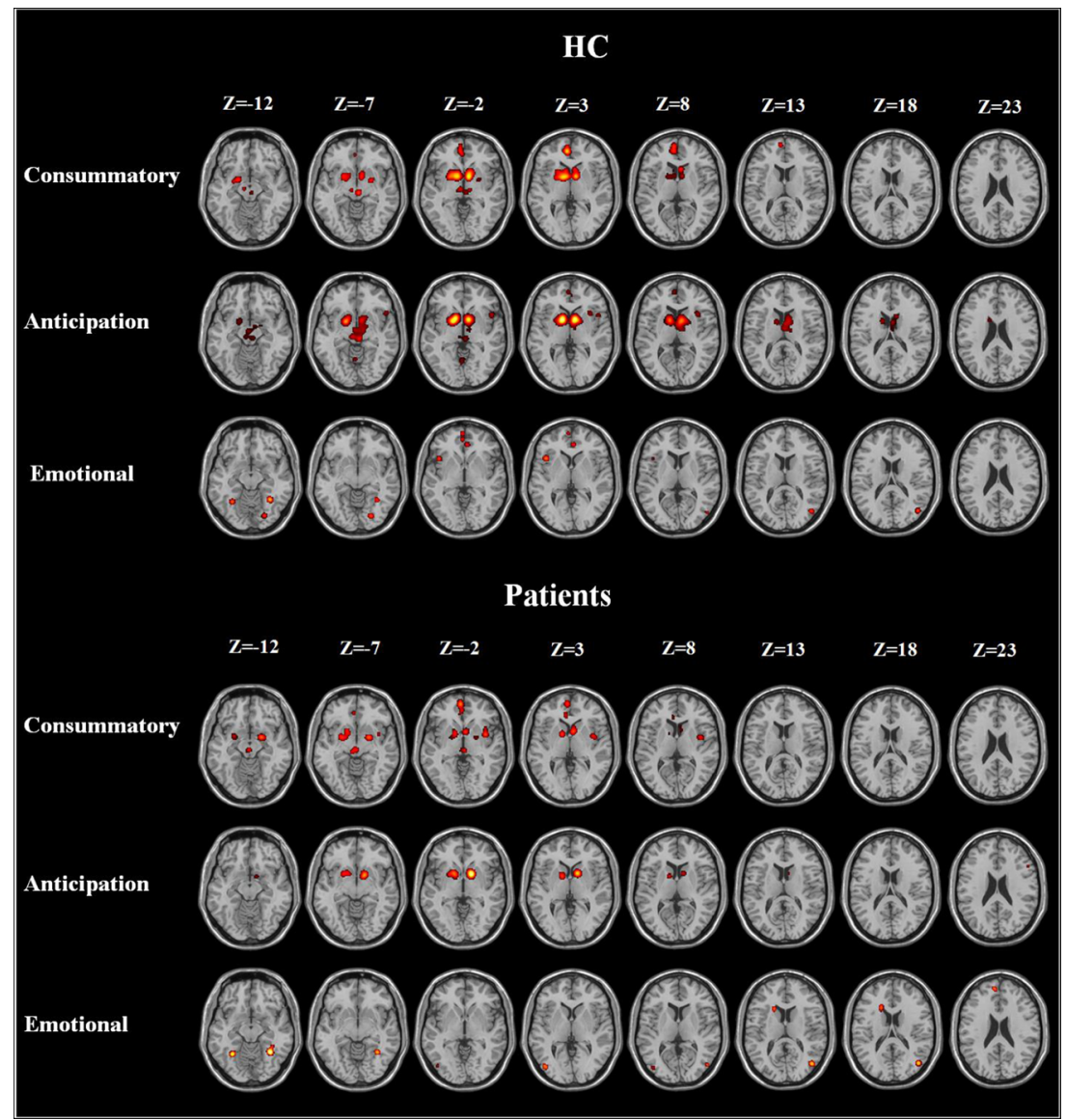


putamen, right hippocampus and parahippocampus, ACC, mPFC and MFG $(p<0.01$, FDR corrected, cluster size $>$ $400 \mathrm{~mm}^{3}$ ). Increased likelihood of activation was observed in the left IFG and MFG.

\section{Emotional processing}

For the emotional experience task (31 studies and 163 foci), decreased likelihood of activation was observed in a number of brain areas from the cortical-subcortical network (Fig. 3 and Table 3), including right GPe, right putamen, right medial dorsal nucleus and ventrolateral nucleus of thalamus, bilateral amygdala, left parahippocampal gyrus, right IFG, left mPFC, left ACC and STG/MTG $(p<0.01$, FDR corrected, cluster size $>400 \mathrm{~mm}^{3}$ ). Increased likelihood of activation was observed in the left middle occipital gyrus and fusiform gyrus.

\section{Between-group ALE analysis in MDD or SZ}

\section{$M D D$}

For consummatory anhedonia in MDD (21 studies and 107 foci), decreased likelihood of activation was observed in the left GPe, right caudate body, left putamen, right insula and left ACC $(p<0.01$, FDR corrected, cluster size $>400 \mathrm{~mm} 3$; Table S2 and Fig. 4). No areas with increased likelihood activation were observed.

For anticipatory anhedonia in MDD (17 studies and 89 foci), decreased likelihood of activation was observed in bilateral caudate head and left MFG, and increased activation was observed in left IFG and right MFG.
For emotional processing in MDD (25 studies and 124 foci), decreased likelihood of activation was observed in right GPe and putamen, bilateral amygdala and anterior lobe, right IFG and left ACC, and increased activation was observed in middle occipital gyrus and fusiform gyrus.

\section{$S Z$}

For consummatory anhedonia in SZ (8 studies and 44 foci), decreased likelihood of activation was observed in left putamen and caudate head, pulvinar and right red nucleus $(p<0.01$, FDR corrected, cluster size $>400 \mathrm{~mm} 3$; Table S3 and Fig. 4). No areas with increased likelihood of activation were observed.

For anticipatory anhedonia in SZ (13 studies and 30 foci) decreased likelihood of activation was observed in left putamen, ACC, and mPFC and right caudate head. No areas with increased likelihood of activation were observed.

For emotional processing in SZ (6 studies and 39 foci), decreased likelihood of activation was observed in the right ventral lateral nucleus. No areas with increased likelihood of activation were observed.

\section{Discussion}

This review used a transdiagnostic, meta-analytic approach (ALE) to explore the neuronal basis of anhedonia across three subdomains: consummatory anhedonia, anticipatory anhedonia and emotional processing. Results revealed that consummatory anhedonia was associated with decreased activation in ventral basal ganglia areas, ventral tegmental area (VTA) and
Fig. 3 Significant ALE maps (FDR corrected, $p<0.01$, cluster size $>400 \mathrm{~mm} 3$ ) of betweengroup analysis in a transdiagnostic approach across MDD and SZ for three subdomains: (a) consummatory anhedonia (b) anticipatory anehdonia and (c) emotional processing. Red and blue areas depict regions with increased and decreased likelihood of activation in the healthy controls than that in patients, respectively

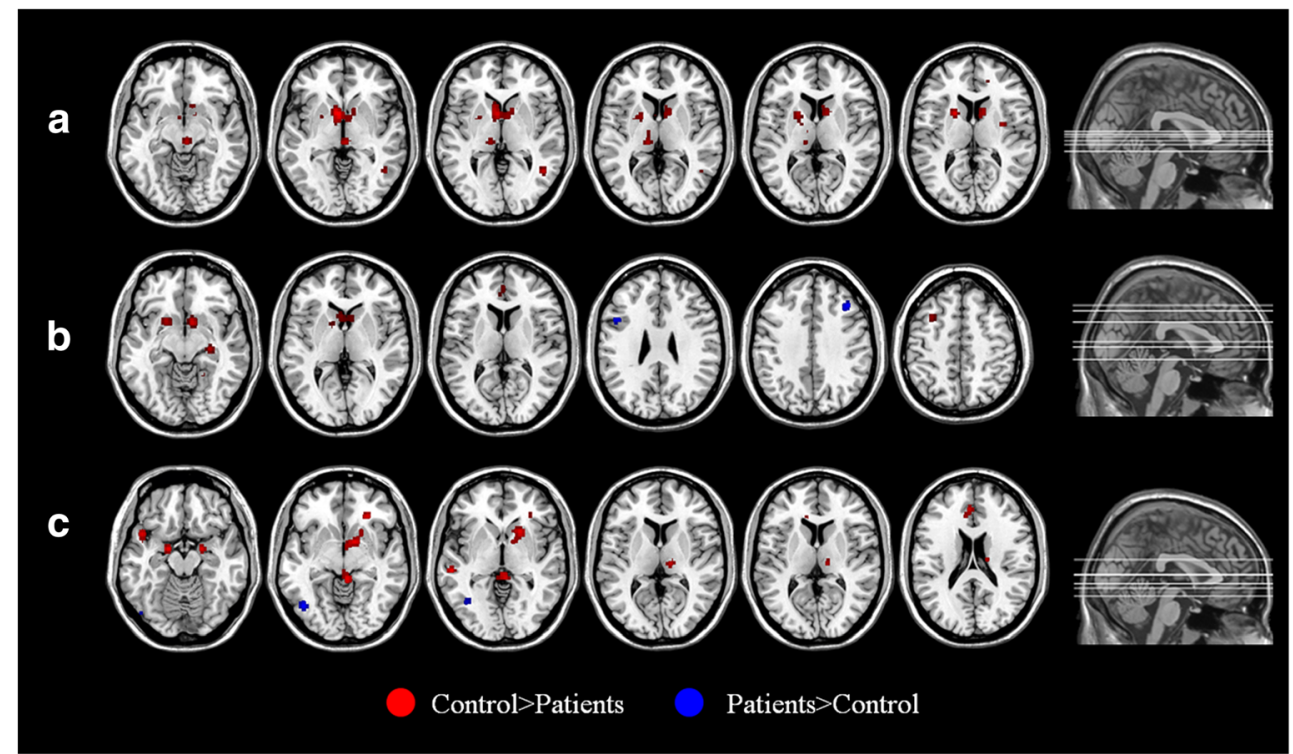


Table 3 ALE results in a transdiagnostic approach across MDD and SZ

\begin{tabular}{|c|c|c|c|c|c|c|c|}
\hline Cluster & Anatomical Region & $\mathrm{BA}$ & $\mathrm{X}$ & $\mathrm{Y}$ & Z & Volume $\mathrm{mm}^{3}$ & Maximum ALE Value $\left(10^{-3}\right)$ \\
\hline \multicolumn{8}{|c|}{ Consummatory anhedonia } \\
\hline \multicolumn{8}{|c|}{ Control $>$ patients } \\
\hline \multirow[t]{5}{*}{1} & L Caudate Head & & -6 & 8 & -2 & 4696 & 21.54 \\
\hline & R Caudate Body & & 12 & 10 & 12 & & 14.07 \\
\hline & R Caudate Head & & 6 & 6 & 2 & & 12.22 \\
\hline & R Lateral Globus Pallidus & & 14 & 6 & -6 & & 11.06 \\
\hline & R Caudate Body & & 10 & 16 & -8 & & 10.26 \\
\hline \multirow[t]{4}{*}{2} & L Putamen & & -20 & 8 & 12 & 1216 & 18.35 \\
\hline & L Putamen & & -26 & 2 & 4 & & 10.70 \\
\hline & L Putamen & & -24 & 4 & -4 & & 9.51 \\
\hline & L Lateral Globus Pallidus & & -18 & -4 & 8 & & 9.25 \\
\hline 3 & R Red Nucleus & & 4 & -22 & -6 & 800 & 18.22 \\
\hline \multirow[t]{2}{*}{4} & L Pulvinar & & -12 & -24 & 4 & 608 & 14.71 \\
\hline & L Ventral Lateral Nucleus & & -10 & -12 & 6 & & 9.73 \\
\hline 5 & R Middle Temporal Gyrus & 37 & 46 & -56 & 2 & 496 & 16.42 \\
\hline \multicolumn{8}{|c|}{ Anticipatory anhedonia } \\
\hline \multicolumn{8}{|c|}{ Control $>$ patients } \\
\hline \multirow[t]{3}{*}{1} & R Caudate Head & & 8 & 12 & -4 & 5248 & 21.63 \\
\hline & L Putamen & & -14 & 8 & -2 & & 21.38 \\
\hline & L Caudate Head & & -4 & 14 & 2 & & 10.62 \\
\hline 2 & R Hippocampus & & 28 & -20 & -8 & 560 & 13.83 \\
\hline \multirow[t]{2}{*}{3} & L Anterior Cingulate & 32 & 0 & 46 & 8 & 560 & 9.84 \\
\hline & L Medial Frontal Gyrus & 10 & -4 & 52 & 14 & & 7.77 \\
\hline 4 & L Middle Frontal Gyrus & 8 & -32 & 14 & 52 & 488 & 11.88 \\
\hline 5 & R Parahippocampal Gyrus & 19 & 22 & -48 & -4 & 464 & 10.95 \\
\hline \multicolumn{8}{|c|}{ Patients $>$ control } \\
\hline 1 & R Middle Frontal Gyrus & 9 & 38 & 26 & 38 & 560 & 13.87 \\
\hline 2 & L Inferior Frontal Gyrus & 9 & -46 & 12 & 30 & 504 & 13.98 \\
\hline \multicolumn{8}{|c|}{ Emotional processing } \\
\hline \multicolumn{8}{|c|}{ Control $>$ patients } \\
\hline \multirow[t]{3}{*}{1} & R Lateral Globus Pallidus & & 14 & 4 & -4 & 2904 & 17.11 \\
\hline & R Amygdala & & 20 & -4 & -12 & & 15.81 \\
\hline & R Putamen & & 20 & 14 & -2 & & 15.73 \\
\hline \multirow[t]{2}{*}{2} & R Anterior Lobe. Culmen & & 6 & -38 & -4 & 1640 & 17.06 \\
\hline & L Parahippocampal Gyrus & 30 & -6 & -38 & 4 & & 11.84 \\
\hline 3 & R Inferior Frontal Gyrus & 47 & 26 & 32 & -8 & 888 & 17.17 \\
\hline \multirow[t]{2}{*}{4} & R Medial Dorsal Nucleus & & 12 & -20 & 6 & 832 & 12.56 \\
\hline & R Ventral Lateral Nucleus & & 14 & -16 & 14 & & 11.42 \\
\hline \multirow[t]{2}{*}{5} & L Medial Frontal Gyrus & 9 & -4 & 40 & 22 & 736 & 12.87 \\
\hline & L Anterior Cingulate & 32 & -10 & 32 & 12 & & 9.50 \\
\hline 6 & L Superior Temporal Gyrus & 38 & -46 & 14 & -12 & 688 & 15.57 \\
\hline 7 & L Amygdala & & -18 & -4 & -14 & 448 & 16.22 \\
\hline 8 & L Middle Temporal Gyrus & 22 & -56 & -28 & 0 & 400 & 15.16 \\
\hline \multicolumn{8}{|c|}{ Patients $>$ control } \\
\hline \multirow[t]{2}{*}{1} & L Middle Occipital Gyrus & 37 & -44 & -66 & -6 & 1160 & 13.15 \\
\hline & L Fusiform Gyrus & 19 & -46 & -74 & -10 & & 9.57 \\
\hline
\end{tabular}

$p<0.01$, FDR corrected, cluster size $>400 \mathrm{~mm}^{3} ; B A=$ Brodmann area 
Fig. 4 Significant ALE maps (FDR corrected, $p<0.01$, cluster size $>400 \mathrm{~mm} 3$ ) of betweengroup analysis in MDD or SZ respectively for three

subdomains: (a) consummatory anehdonia (b) anticipatory anhedonia and (S) emotional processing. Red areas depict the regions with significant different activation between MDD patients and healthy controls. Green areas depict regions with significant different activation between SZ patients and healthy controls. ALE clusters are projected on a standard anatomical template in axial orientation, referring to Talairach space

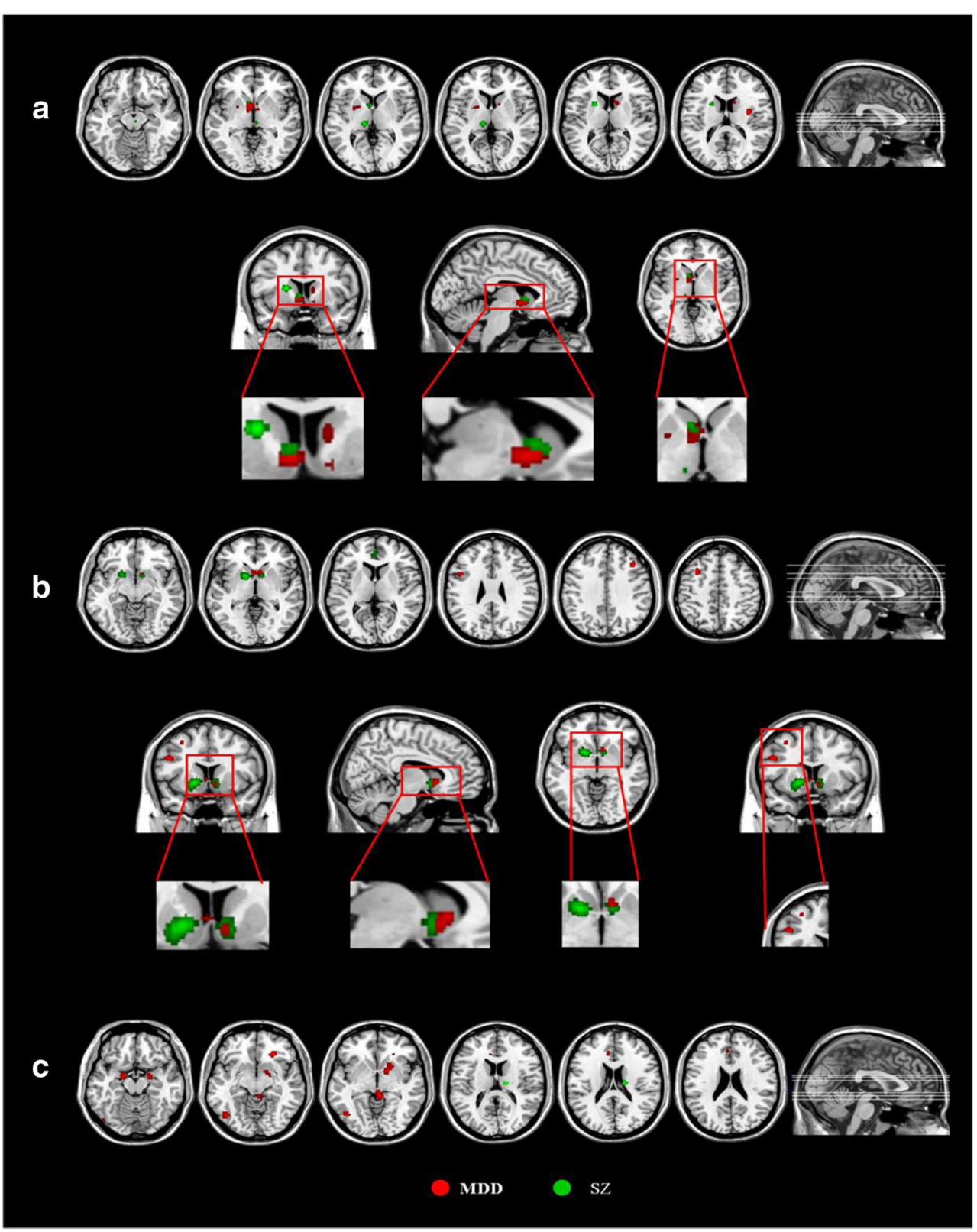

various subcortical areas. Anticipatory anhedonia was related to dysfunctions within basal ganglia regions like caudate and putamen, as well as various subregions of prefrontal cortex, such as anterior cingulate, medial frontal gyrus and middle frontal gyrus. Emotional processing of positive-valence stimuli was related to more distributed dysfunction, involving more of basal ganglia (putamen and GPe), thalamus, limbic regions (amygdala and parahippocampal gyrus), prefrontal cortex (IFG, mPFC, ACC), STG and middle occipital gyrus. Meta-analyses of MDD and SZ respectively revealed similar impairments in brain regions and activation patterns in consummatory and anticipatory anhedonia in these two diseases, but differences in emotional processing.
The neural correlates of different reward processing component

The within-group analysis results of healthy controls showed that the three reward processing components related with anhedonia were differentially recruited the subregions in the basal ganglia and forebrain. Specifically, the reward consummatory processing was mainly concentrated on a series of subcortical regions including GPe, ventral striatum, $\mathrm{SN}$, as well as mPFC and extended orbitofrontal cortex, which is consistent with a number of animal and human studies (Der-Avakian and Markou 2012). Recent researches suggests that the opioid and GABA-ergic systems may play important role in that hedonic 
responses (at least to primary sensory stimuli). On the other side, reward anticipatory processing was more involved in dorsal striatum and cortical areas except for the ventral striatum and medial profrontal areas,, such as inferior frontal gyrus and insula. Those results highlighted importance of frontostriatal circuitry in reward anticipatory/prediction processing (Barch and Dowd 2010; Jarbo and Verstynen 2015) and indicated that further investigation are needed on identifying the connectivites between subareas of frontal cortex and striatum for specific reward component. Unlike the neural correlates of reward consummatory and anticipation, the within-group analyses for emotional processing task revealed much more widely distributed cortical areas in healthy controls, but not many subcortical regions. Those results suggested that the activated brain areas in emotional experience task may be the synthesis of various cognitive and emotional response related with reward. Furthermore, both the transdiagnostic analysis with mixed patients group and the analysis with MDD or SZ group separately showed a similarly contributed but less activated pattern with that of healthy controls in each of the subdomains of anhedonia.

\section{Transdiagnostic neural markers of anhedonia}

In both MDD and SZ, anhedonia is considered to be a core clinical characteristic (Griffiths et al. 2014; Treadway et al. 2012; Wang et al. 2008). Animal studies have demonstrated parallel substrates across different disorders associated with altered anhedonia-related processing (Barr and Phillips 2002; Der-Avakian and Markou 2010). Therefore, researchers suggested that common pathological mechanisms could underlie anhedonia-related processing, supporting a transdiagnostic approach to reveal these underlying cognitive and neurobiological mechanisms. In this review, we applied an ALE meta-analysis to MDD and SZ to provide an integrated framework of the neural bases of anhedonia.

Based on the results of this transdiagnostic meta-analysis, consummatory anhedonia is related to decreased activation in several subcortical regions, such as caudate head and body, putamen, red nucleus, GPe, and pulvinar. Animal studies have demonstrated involvement of the ventral striatum and ventral pallidum in the experience of pleasure and hedonic perception of rewards (Berridge and Kringelbach 2008; Kelley et al. 2002). Furthermore, patients with ventral pallidus damage have significantly reduced responses to rewards or pleasant stimuli (Miller et al. 2006; Vijayaraghavan et al. 2008). Consistent with previous findings, our meta-analysis further implicated the opioid and GABA-ergic systems in the nucleus accumbens shell and its projections to the ventral pallidum, in deficits of in-themoment hedonic experience. Specifically, reduced caudate activation has been reported to be associated with blunted processing of incentive perception and reinforcement learning in both MDD and SZ (Dowd and Barch 2010; Pizzagalli 2014; Pizzagalli et al. 2009). A more recent conceptualization of pulvinar function posited that this region plays an important role in emotional salience and awareness (Hamilton et al. 2012; Pessoa and Adolphs 2010) and has strong bidirectional connectivity with insula and dACC (Mufson and Mesulam 1984). Given this role of the pulvinar, we propose that lowlevel pulvinar activation may blunt hedonic experience through insufficient attention and awareness of reward-related information. To summarize, ALE meta-analysis on consummatory anhedonia processing emphasized the critical role of ventral basal ganglia and medial prefrontal pathways in generating the inthe-moment hedonic value representation and experience.

For anticipatory anhedonia, ALE showed involvement of the caudate and putamen, as well as several prefrontal subregions, such as dACC (BA32), mPFC and MFG. Previous studies suggested that multiple prefrontal-striatal pathways and reciprocal connections within subregions of PFC play an important role in regulating behavioral response to rewards or pleasurable stimuli in the motivational/anticipatory stage (Der-Avakian and Markou 2012; Salamone and Correa 2012). For instance, disruption of glutamatergic signaling between the mPFC and nucleus accumbens resulted in avolition for rewards (Faure et al. 2010). Moreover, striatal lesions or dopamine depletion in striatum or dACC impaired the computation of effort-related costs, which then lead to a deficit of effort-based decision-making as the expected rewards were discounted (Croxson et al. 2009). Preclinical studies further stressed that dACC plays an important role in signaling the net value (benefits minus costs) during social interactions (Apps and Ramnani 2014). In brief, during the anticipation stage for reward or pleasurable stimuli, abnormal activation in $\mathrm{MPFC}$, dACC and MFG may lead to failure to anticipate forthcoming rewards, including updating/maintaining the value of pleasant stimuli, effort-value computation, decision-making to engage in goal-directed activity and monitoring incentive-based behavioral responses (Haber and Knutson 2010; Pujara and Koenigs 2014; Wallis and Kennerley 2010). The results of our meta-analysis consistently indicated that a number of brain regions and pathways, involving many neurotransmitter systems, mediated different aspects of anhedonia-related deficits. Consequently, this preliminary neural framework supports the conceptualization of anhedonia as a symptom with multiple components. Rigorous experimental paradigms and careful designs should be carried out in future work to provide a more refined description of anhedonia and to separate the neural bases of each aspect of anhedonia-related processing.

Our ALE analysis for anticipatory anhedonia also revealed reduced activation of the hippocampus and parahippocampal gyrus, which could impair retrieval of previous autobiographical memories and their incorporation into working memory to represent the value of a reward and anticipate a pleasurable stimulus (Adcock et al. 2006; Zhu et al. 2012; Mazgaj et al. 2015). However, we did not observe any significant group difference between patients and controls in orbitofrontal cortex (OFC), 
which is considered to be a key cortical region involved in anhedonia, in particular in the reward value presentation (Kringelbach 2005; Pizzagalli 2014). One possible explanation is that detection of OFC is challenging for functional MRI experiments using regular EPI sequences due to strong magnetic susceptibility variation near the air-filled sinuses and corresponding signal loss and distortion (Faro et al. 2006; Osterbauer et al. 2006). Future investigation of this unique structure will benefit from advances in imaging sequences or MRI hardware.

Given the complex neural pathways in the frontal-striatalthalamic circuitry, it is necessary to further divide each subcortical structure (e.g., striatum) into subregions to investigate the function of different cortical-subcortical connections. For instance, the striatum can be divided into three functional subregions (i.e., limbic, associative and sensorimotor) based on afferent inputs from the frontal cortex (Haber 2010; Haber et al. 2000; Iversen 2010; van den Bos et al. 2014). Consummatory anhedonia is mainly associated with limbic/ventral striatum, including anterior and ventral caudate and putamen (Corral-Frias et al. 2015; Pizzagalli et al. 2009), whereas anticipatory anhedonia is associated with both limbic/ventral striatum and associative striatum, including the dorsal caudate and anterior part of the dorsal putamen (Delgado 2007; Dichter et al. 2009; Mucci et al. 2015). The results of our meta-analysis provide new evidence that dysfunction in distinct frontal-striatal pathways contributes to the different components of anhedonia-related processing. Based on this preliminary neural framework, further studies are needed to identify the functional and structural connections underlying these components of anhedonia-related processing.

ALE analysis of the emotional experience task revealed more widely distributed differences between patients and controls. In addition to blunted activation in basal ganglia and thalamus, a number of regions in limbic areas, prefrontal cortex, temporal cortex, and cerebellum also showed decreased activation in patients, whereas the visual association cortices showed increased activation. Given that the emotional experience task required no response and may include various cognitive and affective components, it is unclear whether those different activations reflect only the neural bases of anhedonia. Recent resting-state fMRI showed that different neural circuits were engaged in the emotional task-general and emotion task-specific processing (Cole et al. 2014), which would be differently activated by the emotional experience task between controls and patients with affective symptoms. Moreover, functional connectivity analysis demonstrated distinct circuits associated with severity of general affective symptoms (i.e., depression) and anhedonia (Gabbay et al. 2013). Indeed, a set of brain regions, including the anterior and posterior cingulate, $\mathrm{mPFC}$, basal ganglia and visual areas, were activated during passive viewing of positive-valence stimuli, but the ventral striatum and mPFC were the key regions correlated with self-reported anhedonia severity (Epstein et al. 2006; Harvey et al. 2010). Therefore, more elaborate experimental designs will be required to explore the underlying neural bases of general mood-related processing and specific emotional experiences (e.g., hedonia).

\section{Disease-specific findings: MDD and SZ}

To further explore anhedonia-related neural mechanisms and identify potential disease-specific or task-specific confounders, ALE analysis was compared between MDD and SZ patients across the three anhedonia subdomains. In general, anhedonia-related impairment was consistent between MDD and SZ patients across the consummatory and anticipatory stages. Those findings suggest that the neural substrates involved in accurate appraisal of positive stimuli and generation of reward responses, but not the substrates underlying hedonic emotion arousal, are likely to be the core brain areas associated with anhedonia. Furthermore, these data provide a partial explanation for the discordance between self-reported trait pleasure and momentary pleasure in previous behavioral studies: MDD and SZ patients reported normative affective ratings in response to evocative laboratory stimuli, but low positive affect and pleasurable experiences on evaluation of inventories (Osuch et al. 2009; Pizzagalli 2010; Strauss 2013; Treadway and Zald 2011). From a cognitive processing pespective, laboratory-based assessment of consummatory anhedonia may reflect the capacity for hedonic experience, while the patient self-reports in clinical interviews and inventories might reflect both the hedonic experience deficit and the inability to accurately represent incentive experiences. Based on this interpretation, the theoretical definition of anhedonia for research and targeted treatment may need to be modified.

In contrast to the consummatory and anticipatory anhedonia domains, which were similar across MDD and SZ, emotional experience tasks showed striking differences. In MDD patients, decreased activation was much more widely distributed across brain regions, including basal ganglia, amygdala, frontal gyrus and cerebellum; SZ patients only showed decreased activation in thalamus. As previously noted, the emotional experience task requires no response and may reflect other cognitive and affective components. Our results support that the emotional experience task induced both affective/mood-related and anhedonia-specific responses. It is worth noting that only blunted activation in part of ventral basal ganglia was correlated with anhedonia in MDD patients viewing positive valence pictures (Epstein et al. 2006; Mitterschiffthaler et al. 2003; Osuch et al. 2009), which was highly consistent with the correlations between dysfunctional BOLD signals and anhedonia in a consummatory anhedonia task (Gradin et al. 2011; Pizzagalli et al. 2009). Overall, it appears that an emotional experience task, such as passively viewing pictures or words, may not be a pure measure of anhedonia (i.e., consummatory anhedonia) and may capture the affective responses related with general mood, 
which may influence results in populations with prominent depressive symptoms.

\section{Limitations}

Potential limitations of this study should be noted. First of all, ALE technique does have its own limitations. Unlike the meta-analyses in which the complete activation maps are included, the data used in ALE were based solely on reported peak activation coordinates. Thereby ALE could not take into account those studies without any significant clusters reported, which may resulting in a systematic overestimation bias of the results. Besides, the present ALE software cannot conduct the correlation analysis or deal with covariates, thus the only way to consider the covariates was run follow-up analyses, e.g., only on experiments investigating only female subjects or unmedicated patients when considering the gender or medication effect. An additional limitation of the ALE is that it may be unable to assess subtle methodological differences in individual studies such as the thresholding used in the original studies, relative strength of activations/differences between groups and differences in preprocessing steps (Radua and Mataix-Cols 2012). Nevertheless, previous studies suggested that no individual study could significantly bias the results of ALE meta-analyses after including the sample size and number of reported foci into ALE algorithm, changing from fixed-effects to random-effects inference, and revising for multiple comparison correction (Eickhoff et al. 2009; Turkeltaub et al. 2012; Eickhoff et al. 2012; Kollndorfer et al. 2013). In the present study, the robustness of results was further supported by the reasonably coherent findings in within-group ALE analysis of various reward processing components and between-group analyses of subdomains of anhedonia, which were also consistent with previous neurobioglogical studies on animals.

Secondly, given that the patients in 31/35 MDD studies and 22/25 SZ studies were treated in their lifetime by antidepressant or antipsychotics, the drug effects on the brain activated pattern in reward-related processing are potential confounding factors which need to be addressed (Clarke et al. 2014; Boccia et al. 2015). Most antidepressant treatments act on the serotonergic or noradrenergic circuits, but not directly enhance Dopamine (DA) neurotransmission, which plays a significant role in motivation and reward processing (Berridge 2007; Treadway and Zald 2011). However, recent literature showed that the antidepressant mechanism such as increasing serotonin (5-HT) originating from midbrain raphe nuclei (RN), could sequentially decrease the dopamine inhibition and increased ventral striatal activity to reward in humans and animals (Dremencov et al. 2005; Dunlop and Nemeroff 2007; Ossewaarde et al. 2011; El Yacoubi et al. 2011). As for the antipsychotic treatment that directly impacts the DA neurotransmission, evidence showed that the first generation (typical) and second generation (atypical) antipsychotic medications had different effects on the neural correlates of reward/motivation tasks (Juckel et al. 2006a, b; Kirsch et al. 2007; Schlagenhauf et al. 2008). For instance, Kirsch et al. found that patients with typical antipsychotics showed reduced ventral striatal activation, while atypically treated patients showed significantly stronger activation of the right ventral striatum. Those results were further supported by a controlled, longitudinal study (Nielsen et al. 2012a) . The different impact of typical and atypical antipsychotics on reinforcement learning of reward processing were also supported by both behavioral evidences (Beninger et al. 2003; Keri et al. 2005) and neuroimaging data (Insel et al. 2014). Further clinical and basic studies are needed to reveal the underlying mechanism of those effects.

\section{Conclusion and future directions}

In summary, our ALE meta-analysis supported characterization of anhedonia by alterations in reward processing, which contain multiple complicated components and rely on many brain regions within frontal-striatal circuitry. Specifically, consummatory anhedonia was associated with decreased activation in ventral striatum and pallidum, while anticipatory anhedonia was more associated with more substrates in frontal-striatal networks except the ventral striatum, which included the dorsal anterior cingulate, middle frontal gyrus and medial frontal gyrus. However, the emotional experience task (passive viewing of positive pictures or words) revealed mixed findings, including dysfunction in both the anhedonia-related and affective/mood processing regions. Therefore, the transdiagnostic approach holds promise for providing both overall and specific neurobiological frameworks of anhedonia.

Although our meta-analysis presents novel, meaningful evidence regarding the neurobiology of anhedonia, it only distinguished the neural substrates of motivation to pursue rewards and hedonic response to rewards. Future work is needed to dissect the different neurobiological pathways that are related to the various reward-processing subcomponents, such as perceiving pleasure, encoding reward value, calculating costs and benefits, learning from prior reinforcement, making decisions and execute the action to pursue a reward. It is important to note that anhedonia also plays an important role in several other psychiatric and neurological disorders, such as Parkinson's disease (Loas et al. 2012), post-traumatic stress disorder (Frewen et al. 2012) and drug addiction (Balodis and Potenza 2015; Hatzigiakoumis et al. 2011), albeit in heterogeneous ways. Therefore, combining more categories of disorders and using a greater sample size in future studies should further elucidate the neural foundations of anhedonia.

Acknowledgments This work was supported by grants from the National Natural Science Foundation of China (30700236 to XW; 61473221 to PL), the Program for New Century Excellent Talents in University of 
China (NCET-12-0557 to XW), the Humanities and social science research project of Ministry of education in China (13YJA190015 to XW), the National Institutes of Health (R01MH094594 to DÖ and K23MH097786 to RPA).

\section{Compliance with ethical standards}

Conflict of interest Dr. Öngür has served on a Scientific Advisory Board for Lilly Inc. in 2013. Author Bei Zhang, Author Pan Lin, Author Huqing Shi, Author Randy P Auerbach, Author Xiaosheng Wang, Author Shuqiao Yao and Author Xiang Wang declare that they have no conflict of interest.

Informed consent No animal or human studies were carried out by the authors for this article.

Open Access This article is distributed under the terms of the Creative Commons Attribution 4.0 International License (http:// creativecommons.org/licenses/by/4.0/), which permits unrestricted use, distribution, and reproduction in any medium, provided you give appropriate credit to the original author(s) and the source, provide a link to the Creative Commons license, and indicate if changes were made.

\section{References}

Adcock, R. A., Thangavel, A., Whitfield-Gabrieli, S., Knutson, B., \& Gabrieli, J. D. (2006). Reward-motivated learning: Mesolimbic activation precedes memory formation. Neuron, 50(3), 507-517.

Admon, R., Nickerson, L. D., Dillon, D. G., Holmes, A. J., Bogdan, R., Kumar, P., et al. (2015). Dissociable cortico-striatal connectivity abnormalities in major depression in response to monetary gains and penalties. Psychological Medicine, 45(1), 121-131.

Apps, M. A., \& Ramnani, N. (2014). The anterior cingulate gyrus signals the net value of others' rewards. Journal of Neuroscience, 34(18), 6190-6200

Association, American Psychological, Association, American Psychological. (1994). Diagnostic and statistical manual of mental disorders: Dsm-iv: American Psychiatric Association.

Balodis, I. M., \& Potenza, M. N. (2015). Anticipatory reward processing in addicted populations: A focus on the monetary incentive delay task. Biological Psychiatry, 77(5), 434-444.

Barch, D. M., \& Dowd, E. C. (2010). Goal representations and motivational drive in schizophrenia: The role of prefrontal-striatal interactions. Schizophrenia Bulletin, 36(5), 919-934.

Barr, A. M., \& Phillips, A. G. (2002). Increased successive negative contrast in rats withdrawn from an escalating-dose schedule of damphetamine. Pharmacology Biochemistry and Behavior, 71(1), 293-299.

Beninger, R. J., Wasserman, J., Zanibbi, K., Charbonneau, D., Mangels, J., \& Beninger, B. V. (2003). Typical and atypical antipsychotic medications differentially affect two nondeclarative memory tasks in schizophrenic patients: A double dissociation. Schizophrenia Research, 61(2-3), 281-292.

Bermpohl, F., Walter, M., Sajonz, B., Lucke, C., Hagele, C., Sterzer, P., et al. (2009). Attentional modulation of emotional stimulus processing in patients with major depression-alterations in prefrontal cortical regions. Neuroscience Letters, 463(2), 108-113.

Berridge, K. C. (2007). The debate over dopamine's role in reward: The case for incentive salience. Psychopharmacology, 191(3), 391-431.

Berridge, K. C., \& Kringelbach, M. L. (2008). Affective neuroscience of pleasure: Reward in humans and animals. Psychopharmacology, 199(3), 457-480.
Berridge, K. C., \& Robinson, T. E. (2003). Parsing reward. Trends in Neurosciences, 26(9), 507-513.

Boccia, M., Piccardi, L., Guariglia, P. (2015). How treatment affects the brain: Meta-analysis evidence of neural substrates underpinning drug therapy and psychotherapy in major depression. Brain Imaging Behav.

Canli, T., Sivers, H., Thomason, M. E., Whitfield-Gabrieli, S., Gabrieli, J. D., \& Gotlib, I. H. (2004). Brain activation to emotional words in depressed vs healthy subjects. Neuroreport, 15(17), 2585-2588.

Chantiluke, K., Halari, R., Simic, M., Pariante, C. M., Papadopoulos, A., Giampietro, V., et al. (2012). Fronto-striato-cerebellar dysregulation in adolescents with depression during motivated attention. Biological Psychiatry, 71(1), 59-67.

Choi, S. H., Lee, H., Ku, J., Yoon, K. J., \& Kim, J. J. (2014). Neural basis of anhedonia as a failure to predict pleasantness in schizophrenia. World Journal of Biological Psychiatry, 15(7), 525-533.

Clarke, H. F., Cardinal, R. N., Rygula, R., Hong, Y. T., Fryer, T. D., Sawiak, S. J., et al. (2014). Orbitofrontal dopamine depletion upregulates caudate dopamine and alters behavior via changes in reinforcement sensitivity. Journal of Neuroscience, 34(22), 7663 7676.

Cole, M. W., Bassett, D. S., Power, J. D., Braver, T. S., \& Petersen, S. E. (2014). Intrinsic and task-evoked network architectures of the human brain. Neuron, 83(1), 238-251.

Corral-Frias, N. S., Nikolova, Y. S., Michalski, L. J., Baranger, D. A., Hariri, A. R., \& Bogdan, R. (2015). Stress-related anhedonia is associated with ventral striatum reactivity to reward and transdiagnostic psychiatric symptomatology. Psychological Medicine, 1-13.

Croxson, P. L., Walton, M. E., O'Reilly, J. X., Behrens, T. E., \& Rushworth, M. F. (2009). Effort-based cost-benefit valuation and the human brain. Journal of Neuroscience, 29(14), 4531-4541.

Cuthbert, B. N., \& Insel, T. R. (2013). Toward the future of psychiatric diagnosis: the seven pillars of rdoc. BMC Medicine, 11(1), 126.

da Silva Alves, F., Bakker, G., Schmitz, N., Abeling, N., Hasler, G., van der Meer, J., et al. (2013). Dopaminergic modulation of the reward system in schizophrenia: a placebo-controlled dopamine depletion fmri study. European Neuropsychopharmacology, 23(11), 15771586.

Delgado, M. R. (2007). Reward-related responses in the human striatum. Annals of the New York Academy of Sciences, 1104, 70-88.

Der-Avakian, A., \& Markou, A. (2010). Withdrawal from chronic exposure to amphetamine, but not nicotine, leads to an immediate and enduring deficit in motivated behavior without affecting social interaction in rats. Behavioural Pharmacology, 21(4), 359.

Der-Avakian, A., \& Markou, A. (2012). The neurobiology of anhedonia and other reward-related deficits. Trends in Neurosciences, 35(1), 68-77.

Derntl, B., Seidel, E. M., Eickhoff, S. B., Kellermann, T., Gur, R. C., Schneider, F., et al. (2011). Neural correlates of social approach and withdrawal in patients with major depression. Social Neuroscience, 6(5-6), 482-501.

Dichter, G. S., Felder, J. N., Petty, C., Bizzell, J., Ernst, M., \& Smoski, M. J. (2009). The effects of psychotherapy on neural responses to rewards in major depression. Biological Psychiatry, 66(9), 886-897.

Dichter, G. S., Kozink, R. V., McClernon, F. J., \& Smoski, M. J. (2012). Remitted major depression is characterized by reward network hyperactivation during reward anticipation and hypoactivation during reward outcomes. Journal of Affective Disorders, 136(3), 11261134.

Dillon, D. G., Holmes, A. J., Jahn, A. L., Bogdan, R., Wald, L. L., \& Pizzagalli, D. A. (2008). Dissociation of neural regions associated with anticipatory versus consummatory phases of incentive processing. Psychophysiology, 45(1), 36-49.

Dowd, E. C., \& Barch, D. M. (2010). Anhedonia and emotional experience in schizophrenia: neural and behavioral indicators. Biological Psychiatry, 67(10), 902-911. 
Dowd, E. C., \& Barch, D. M. (2012). Pavlovian reward prediction and receipt in schizophrenia: relationship to anhedonia. PloS One, 7(5), e35622.

Dremencov, E., Newman, M. E., Kinor, N., Blatman-Jan, G., Schindler, C. J., Overstreet, D. H., et al. (2005). Hyperfunctionality of serotonin-2c receptor-mediated inhibition of accumbal dopamine release in an animal model of depression is reversed by antidepressant treatment. Neuropharmacology, 48(1), 34-42.

Dunlop, B. W., \& Nemeroff, C. B. (2007). The role of dopamine in the pathophysiology of depression. Archives of General Psychiatry, 64(3), 327-337.

Eickhoff, S. B., Laird, A. R., Grefkes, C., Wang, L. E., Zilles, K., \& Fox, P. T. (2009). Coordinate-based activation likelihood estimation meta-analysis of neuroimaging data: a random-effects approach based on empirical estimates of spatial uncertainty. Human Brain Mapping, 30(9), 2907-2926.

Eickhoff, S. B., Bzdok, D., Laird, A. R., Kurth, F., \& Fox, P. T. (2012). Activation likelihood estimation meta-analysis revisited. NeuroImage, 59(3), 2349-2361.

El Yacoubi, M., Dubois, M., Gabriel, C., Mocaer, E., \& Vaugeois, J. M. (2011). Chronic agomelatine and fluoxetine induce antidepressantlike effects in $\mathrm{h} /$ Rouen mice, a genetic mouse model of depression. Pharmacology Biochemistry and Behavior, 100(2), 284-288.

Elman, I., Lowen, S., Frederick, B. B., Chi, W., Becerra, L., \& Pitman, R. K. (2009). Functional neuroimaging of reward circuitry responsivity to monetary gains and losses in posttraumatic stress disorder. Biological Psychiatry, 66(12), 1083-1090.

Epstein, J., Pan, H., Kocsis, J. H., Yang, Y., Butler, T., Chusid, J., et al. (2006). Lack of ventral striatal response to positive stimuli in depressed versus normal subjects. The American Journal of Psychiatry, 163(10).

Esslinger, C., Englisch, S., Inta, D., Rausch, F., Schirmbeck, F., Mier, D., et al. (2012). Ventral striatal activation during attribution of stimulus saliency and reward anticipation is correlated in unmedicated first episode schizophrenia patients. Schizophrenia Research, 140(1-3), 114-121.

Faro, Scott H, Mohamed, Feroze B. (2006). Functional mri: Basic principles and clinical applications: Springer Science \& Business Media.

Faure, A., Richard, J. M., \& Berridge, K. C. (2010). Desire and dread from the nucleus accumbens: cortical glutamate and subcortical gaba differentially generate motivation and hedonic impact in the rat. PloS One, 5(6), e11223.

Fervaha, G., Graff-Guerrero, A., Zakzanis, K. K., Foussias, G., Agid, O., \& Remington, G. (2013). Incentive motivation deficits in schizophrenia reflect effort computation impairments during cost-benefit decision-making. Journal of Psychiatric Research, 47(11), 1590 1596.

Forbes, E. E., Hariri, A. R., Martin, S. L., Silk, J. S., Moyles, D. L., Fisher, P. M., et al. (2009). Altered striatal activation predicting real-world positive affect in adolescent major depressive disorder. The American Journal of Psychiatry, 166(1), 64-73.

Fournier, J. C., Keener, M. T., Mullin, B. C., Hafeman, D. M., Labarbara, E. J., Stiffler, R. S., et al. (2013). Heterogeneity of amygdala response in major depressive disorder: the impact of lifetime subthreshold mania. Psychological Medicine, 43(2), 293-302.

Frewen, P. A., Dozois, D. J., Lanius, R. A. (2012). Assessment of anhedonia in psychological trauma: Psychometric and neuroimaging perspectives. Eur J Psychotraumatol, 3.

Fu, C. H., Williams, S. C., Brammer, M. J., Suckling, J., Kim, J., Cleare, A. J., et al. (2007). Neural responses to happy facial expressions in major depression following antidepressant treatment. The American Journal of Psychiatry, 164(4), 599-607.

Gabbay, V., Ely, B. A., Li, Q., Bangaru, S. D., Panzer, A. M., Alonso, C. M., et al. (2013). Striatum-based circuitry of adolescent depression and anhedonia. Journal of the American Academy of Child and Adolescent Psychiatry, 52(6), 628-641. e613.

Gold, J. M., Waltz, J. A., Prentice, K. J., Morris, S. E., \& Heerey, E. A. (2008). Reward processing in schizophrenia: a deficit in the representation of value. Schizophrenia Bulletin, 34(5), 835-847.

Gorka, S. M., Huggins, A. A., Fitzgerald, D. A., Nelson, B. D., Phan, K. L., \& Shankman, S. A. (2014). Neural response to reward anticipation in those with depression with and without panic disorder. Journal of Affective Disorders, 164, 50-56.

Gotlib, I. H., Sivers, H., Gabrieli, J. D., Whitfield-Gabrieli, S., Goldin, P., Minor, K. L., et al. (2005). Subgenual anterior cingulate activation to valenced emotional stimuli in major depression. Neuroreport, 16(16), 1731-1734.

Gotlib, I. H., Hamilton, J. P., Cooney, R. E., Singh, M. K., Henry, M. L., \& Joormann, J. (2010). Neural processing of reward and loss in girls at risk for major depression. Archives of General Psychiatry, 67(4), 380-387.

Gradin, V. B., Kumar, P., Waiter, G., Ahearn, T., Stickle, C., Milders, M., et al. (2011). Expected value and prediction error abnormalities in depression and schizophrenia. Brain, 134, 1751-1764.

Griffiths, K. R., Morris, R. W., \& Balleine, B. W. (2014). Translational studies of goal-directed action as a framework for classifying deficits across psychiatric disorders. Frontiers in Systems Neuroscience, 8.

Grimm, O., Vollstadt-Klein, S., Krebs, L., Zink, M., \& Smolka, M. N. (2012). Reduced Striatal activation during reward anticipation due to appetite-provoking cues in chronic schizophrenia: a fmri study. Schizophrenia Research, 134(2-3), 151-157.

Haber, S. N. (2010). convergence of limbic, cognitive, and motor corticostriatal circuits with dopamine pathways in primate brain. Dopamine handbook, 38 .

Haber, S. N., \& Knutson, B. (2010). The reward circuit: linking primate anatomy and human imaging. Neuropsychopharmacology, 35(1), 4-26.

Haber, S. N., Fudge, J. L., \& McFarland, N. R. (2000). Striatonigrostriatal pathways in primates form an ascending spiral from the shell to the dorsolateral striatum. The Journal of Neuroscience, 20(6), 23692382

Hall, G. B., Milne, A. M., \& Macqueen, G. M. (2014). An fmri study of reward circuitry in patients with minimal or extensive history of major depression. European Archives of Psychiatry and Clinical Neuroscience, 264(3), 187-198.

Hamilton, J. P., Etkin, A., Furman, D. J., Lemus, M. G., Johnson, R. F., \& Gotlib, I. H. (2012). Functional neuroimaging of major depressive disorder: a meta-analysis and new integration of base line activation and neural response data. The American Journal of Psychiatry, 169(7), 693-703.

Harvey, P. O., Armony, J., Malla, A., \& Lepage, M. (2010). Functional neural substrates of self-reported physical anhedonia in non-clinical individuals and in patients with schizophrenia. Journal of Psychiatric Research, 44(11), 707-716.

Hatzigiakoumis, D. S., Martinotti, G., Giannantonio, M. D., \& Janiri, L. (2011). Anhedonia and substance dependence: clinical correlates and treatment options. Front Psychiatry, 2, 10.

Hooker, C. I., Benson, T. L., Gyurak, A., Yin, H., Tully, L. M., \& Lincoln, S. H. (2014). Neural activity to positive expressions predicts daily experience of schizophrenia-spectrum symptoms in adults with high social anhedonia. Journal of Abnormal Psychology, 123(1), 190 204.

Insel, T., Cuthbert, B., Garvey, M., Heinssen, R., Pine, D. S., Quinn, K., et al. (2010). Research domain criteria (rdoc): toward a new classification framework for research on mental disorders. The American Journal of Psychiatry, 167(7), 748-751.

Insel, C., Reinen, J., Weber, J., Wager, T. D., Jarskog, L. F., Shohamy, D., et al. (2014). Antipsychotic dose modulates behavioral and neural responses to feedback during reinforcement learning in 
schizophrenia. Cognitive, Affective, \& Behavioral Neuroscience, 14(1), 189-201.

Iversen, Leslie L. (2010). Dopamine handbook: Oxford University Press.

Jarbo, K., \& Verstynen, T. D. (2015). Converging structural and functional connectivity of orbitofrontal, dorsolateral prefrontal, and posterior parietal cortex in the human striatum. Journal of Neuroscience, 35(9), 3865-3878

Jensen, J., Willeit, M., Zipursky, R. B., Savina, I., Smith, A. J., Menon, M., et al. (2008). The formation of abnormal associations in schizophrenia: neural and behavioral evidence. Neuropsychopharmacology, 33(3), 473-479.

Johnston, B. A., Tolomeo, S., Gradin, V., Christmas, D., Matthews, K., Douglas Steele, J. (2015). Failure of hippocampal deactivation during loss events in treatment-resistant depression. Brain.

Juckel, G., Schlagenhauf, F., Koslowski, M., Filonov, D., Wustenberg, T., Villringer, A., et al. (2006a). Dysfunction of ventral striatal reward prediction in schizophrenic patients treated with typical, not atypical, neuroleptics. Psychopharmacology, 187(2), 222-228.

Juckel, G., Schlagenhauf, F., Koslowski, M., Wustenberg, T., Villringer, A., Knutson, B., et al. (2006b). Dysfunction of ventral striatal reward prediction in schizophrenia. NeuroImage, 29(2), 409-416.

Keedwell, P. A., Andrew, C., Williams, S. C., Brammer, M. J., \& Phillips, M. L. (2005). A double dissociation of ventromedial prefrontal cortical responses to sad and happy stimuli in depressed and healthy individuals. Biological Psychiatry, 58(6), 495-503.

Kelley, A. E., Bakshi, V. P., Haber, S. N., Steininger, T. L., Will, M. J., \& Zhang, M. (2002). Opioid modulation of taste hedonics within the ventral striatum. Physiology \& Behavior, 76(3), 365-377.

Keri, S., Nagy, O., Kelemen, O., Myers, C. E., \& Gluck, M. A. (2005). Dissociation between medial temporal lobe and basal ganglia memory systems in schizophrenia. Schizophrenia Research, 77(2-3), 321-328.

Kirsch, P., Ronshausen, S., Mier, D., \& Gallhofer, B. (2007). The influence of antipsychotic treatment on brain reward system reactivity in schizophrenia patients. Pharmacopsychiatry, 40(5), 196-198.

Knutson, B., Bhanji, J. P., Cooney, R. E., Atlas, L. Y., \& Gotlib, I. H. (2008). Neural responses to monetary incentives in major depression. Biological Psychiatry, 63(7), 686-692.

Koch, K., Schachtzabel, C., Wagner, G., Schikora, J., Schultz, C., Reichenbach, J. R., et al. (2010). Altered activation in association with reward-related trial-and-error learning in patients with schizophrenia. NeuroImage, 50(1), 223-232.

Kollndorfer, K., Krajnik, J., Woitek, R., Freiherr, J., Prayer, D., \& Schopf, V. (2013). Altered likelihood of brain activation in attention and working memory networks in patients with multiple sclerosis: an ale meta-analysis. Neuroscience and Biobehavioral Reviews, 37(10 Pt 2), 2699-2708.

Kringelbach, M. L. (2005). The human orbitofrontal cortex: Linking reward to hedonic experience. Nature Reviews Neuroscience, 6(9), 691-702.

Kumar, P., Waiter, G., Ahearn, T., Milders, M., Reid, I., \& Steele, J. D. (2008). Abnormal temporal difference reward-learning signals in major depression. Brain, 131(Pt 8), 2084-2093.

Kumari, V., Mitterschiffthaler, M. T., Teasdale, J. D., Malhi, G. S., Brown, R. G., Giampietro, V., et al. (2003). Neural abnormalities during cognitive generation of affect in treatment-resistant depression. Biological Psychiatry, 54(8), 777-791.

Laird, A. R., Fox, P. M., Price, C. J., Glahn, D. C., Uecker, A. M., Lancaster, J. L., et al. (2005). Ale meta-analysis: controlling the false discovery rate and performing statistical contrasts. Human Brain Mapping, 25(1), 155-164.

Leventhal, A. M., Chasson, G. S., Tapia, E., Miller, E. K., \& Pettit, J. W. (2006). Measuring hedonic capacity in depression: a psychometric analysis of three anhedonia scales. Journal of Clinical Psychology, 62(12), 1545-1558.
Loas, G., Krystkowiak, P., \& Godefroy, O. (2012). Anhedonia in parkinson's disease: an overview. Journal of Neuropsychiatry and Clinical Neurosciences, 24(4), 444-451.

Markou, A., Chiamulera, C., Geyer, M. A., Tricklebank, M., \& Steckler, T. (2009). Removing obstacles in neuroscience drug discovery: the future path for animal models. Neuropsychopharmacology, 34(1), $74-89$.

Mazgaj, R., Tal, A., Goetz, R., Lazar, M., Rothman, K., Messinger, J. W., et al. (2015). Hypo-metabolism of the rostral anterior cingulate cortex associated with working memory impairment in 18 cases of schizophrenia. Brain Imaging Behav.

McCabe, C., Cowen, P. J., \& Harmer, C. J. (2009). Neural representation of reward in recovered depressed patients. Psychopharmacology, 205(4), 667-677.

Miller, J. M., Vorel, S. R., Tranguch, A. J., Kenny, E. T., Mazzoni, P., van Gorp, W. G., et al. (2006). Anhedonia after a selective bilateral lesion of the globus pallidus. American Journal of Psychiatry, 163(5), 786-788.

Mitterschiffthaler, M. T., Kumari, V., Malhi, G. S., Brown, R. G., Giampietro, V. P., Brammer, M. J., et al. (2003). Neural response to pleasant stimuli in anhedonia: an fmri study. Neuroreport, 14(2), $177-182$.

Morris, R. W., Vercammen, A., Lenroot, R., Moore, L., Langton, J. M., Short, B., et al. (2012). Disambiguating ventral striatum fmri-related bold signal during reward prediction in schizophrenia. Molecular Psychiatry, 17(3), 235, 280-239.

Mucci, A., Dima, D., Soricelli, A., Volpe, U., Bucci, P., Frangou, S., et al. (2015). Is avolition in schizophrenia associated with a deficit of dorsal caudate activity? A functional magnetic resonance imaging study during reward anticipation and feedback. Psychological medicine, 1-14.

Mufson, E. J., \& Mesulam, M. M. (1984). Thalamic connections of the insula in the rhesus monkey and comments on the paralimbic connectivity of the medial pulvinar nucleus. Journal of Comparative Neurology, 227(1), 109-120.

Murray, G. K., Corlett, P. R., Clark, L., Pessiglione, M., Blackwell, A. D., Honey, G., et al. (2008). Substantia nigra/ventral tegmental reward prediction error disruption in psychosis. Molecular Psychiatry, 13(3), 267-276

Nielsen, M. O., Rostrup, E., Wulff, S., Bak, N., Broberg, B. V., Lublin, H., et al. (2012a). Improvement of brain reward abnormalities by antipsychotic monotherapy in schizophrenia. Archives of General Psychiatry, 69(12), 1195-1204.

Nielsen, M. O., Rostrup, E., Wulff, S., Bak, N., Lublin, H., Kapur, S., et al. (2012b). Alterations of the brain reward system in antipsychotic naive schizophrenia patients. Biological Psychiatry, 71(10), 898905.

Ossewaarde, L., Verkes, R. J., Hermans, E. J., Kooijman, S. C., Urner, M., Tendolkar, I., et al. (2011). Two-week administration of the combined serotonin-noradrenaline reuptake inhibitor duloxetine augments functioning of mesolimbic incentive processing circuits. Biological Psychiatry, 70(6), 568-574.

Osterbauer, R. A., Wilson, J. L., Calvert, G. A., \& Jezzard, P. (2006). Physical and physiological consequences of passive intra-oral shimming. NeuroImage, 29(1), 245-253.

Osuch, E. A., Bluhm, R. L., Williamson, P. C., Theberge, J., Densmore, M., \& Neufeld, R. W. J. (2009). Brain activation to favorite music in healthy controls and depressed patients. Neuroreport, 20(13), 12041208

Pessoa, L., \& Adolphs, R. (2010). Emotion processing and the amygdala: from a'low road'to 'many roads' of evaluating biological significance. Nature Reviews Neuroscience, 11(11), 773-783.

Pizzagalli, D. A. (2010). The "anhedonia paradox" in schizophrenia: insights from affective neuroscience. Biological Psychiatry, 67(10), 899-901. 
Pizzagalli, D. A. (2014). Depression, stress, and anhedonia: toward a synthesis and integrated model. Annual Review of Clinical Psychology, 10, 393-423.

Pizzagalli, D. A., Iosifescu, D., Hallett, L. A., Ratner, K. G., \& Fava, M. (2008). Reduced hedonic capacity in major depressive disorder: evidence from a probabilistic reward task. Journal of Psychiatric Research, 43(1), 76-87.

Pizzagalli, D. A., Holmes, A. J., Dillon, D. G., Goetz, E. L., Birk, J. L., Bogdan, R., et al. (2009). Reduced caudate and nucleus accumbens response to rewards in unmedicated individuals with major depressive disorder. The American Journal of Psychiatry, 166(6), 702710 .

Pujara, M., \& Koenigs, M. (2014). Mechanisms of reward circuit dysfunction in psychiatric illness: prefrontal-striatal interactions. The Neuroscientist, 20(1), 82-95.

Radua, J., \& Mataix-Cols, D. (2012). Meta-analytic methods for neuroimaging data explained. Biology Mood Anxiety Disorders, 2(1), 6.

Remijnse, P. L., Nielen, M. M., van Balkom, A. J., Hendriks, G. J., Hoogendijk, W. J., Uylings, H. B., et al. (2009). Differential frontal-striatal and paralimbic activity during reversal learning in major depressive disorder and obsessive-compulsive disorder. Psychological Medicine, 39(9), 1503-1518.

Reske, M., Kellermann, T., Habel, U., Jon Shah, N., Backes, V., von Wilmsdorff, M., et al. (2007). Stability of emotional dysfunctions? A long-term fmri study in first-episode schizophrenia. Journal of Psychiatric Research, 41(11), 918-927.

Robinson, O. J., Cools, R., Carlisi, C. O., Sahakian, B. J., \& Drevets, W. C. (2012). Ventral striatum response during reward and punishment reversal learning in unmedicated major depressive disorder. The American Journal of Psychiatry, 169(2), 152-159.

Rotge, J. Y., Guehl, D., Dilharreguy, B., Tignol, J., Bioulac, B., Allard, M., et al. (2009). Meta-analysis of brain volume changes in obsessive-compulsive disorder. Biological Psychiatry, 65(1), 75-83.

Salamone, J. D., \& Correa, M. (2012). The mysterious motivational functions of mesolimbic dopamine. Neuron, 76(3), 470-485.

Schlagenhauf, F., Juckel, G., Koslowski, M., Kahnt, T., Knutson, B., Dembler, T., et al. (2008). Reward system activation in schizophrenic patients switched from typical neuroleptics to olanzapine. Psychopharmacology, 196(4), 673-684.

Schlagenhauf, F., Sterzer, P., Schmack, K., Ballmaier, M., Rapp, M., Wrase, J., et al. (2009). Reward feedback alterations in unmedicated schizophrenia patients: relevance for delusions. Biological Psychiatry, 65(12), 1032-1039.

Shi, H., Wang, X., \& Yao, S. (2013). Comparison of activation patterns between masking and inattention tasks: a coordinate-based metaanalysis of implicit emotional face processing. Frontiers in Human Neuroscience, 7, 459.

Simon, J. J., Biller, A., Walther, S., Roesch-Ely, D., Stippich, C., Weisbrod, M., et al. (2010). Neural correlates of reward processing in schizophrenia - relationship to apathy and depression. Schizophrenia Research, 118(1-3), 154-161.

Smoski, M. J., Felder, J., Bizzell, J., Green, S. R., Ernst, M., Lynch, T. R., et al. (2009). Fmri of alterations in reward selection, anticipation, and feedback in major depressive disorder. Journal of Affective Disorders, 118(1-3), 69-78.

Smoski, M. J., Rittenberg, A., \& Dichter, G. S. (2011). Major depressive disorder is characterized by greater reward network activation to monetary than pleasant image rewards. Psychiatry ResearchNeuroimaging, 194(3), 263-270.

Steele, J. D., Kumar, P., \& Ebmeier, K. P. (2007). Blunted response to feedback information in depressive illness. Brain, 130(Pt 9), 2367 2374.

Stoy, M., Schlagenhauf, F., Sterzer, P., Bermpohl, F., Hagele, C., Suchotzki, K., et al. (2012). Hyporeactivity of ventral striatum towards incentive stimuli in unmedicated depressed patients normalizes after treatment with escitalopram. Journal of Psychopharmacology, 26(5), 677-688.

Strauss, Gregory P. (2013). The emotion paradox of anhedonia in schizophrenia: Or is it? Schizophrenia bulletin, sbs192.

Stuhrmann, A., Dohm, K., Kugel, H., Zwanzger, P., Redlich, R., Grotegerd, D., et al. (2013). Mood-congruent amygdala responses to subliminally presented facial expressions in major depression: associations with anhedonia. Journal of Psychiatry \& Neuroscience, 38(4), 249-258.

Surguladze, S., Brammer, M. J., Keedwell, P., Giampietro, V., Young, A. W., Travis, M. J., et al. (2005). A differential pattern of neural response toward sad versus happy facial expressions in major depressive disorder. Biological Psychiatry, 57(3), 201-209.

Treadway, M. T., \& Zald, D. H. (2011). Reconsidering anhedonia in depression: lessons from translational neuroscience. Neuroscience \& Biobehavioral Reviews, 35(3), 537-555.

Treadway, M. T., Buckholtz, J. W., Schwartzman, A. N., Lambert, W. E., \& Zald, D. H. (2009). Worth the 'eefrt'? The effort expenditure for rewards task as an objective measure of motivation and anhedonia. Plos One, 4(8).

Treadway, M. T., Bossaller, N. A., Shelton, R. C., \& Zald, D. H. (2012). Effort-based decision-making in major depressive disorder: a translational model of motivational anhedonia. Journal of Abnormal Psychology, 121(3), 553-558.

Tremblay, L. K., Naranjo, C. A., Graham, S. J., Herrmann, N., Mayberg, H. S., Hevenor, S., et al. (2005). Functional neuroanatomical substrates of altered reward processing in major depressive disorder revealed by a dopaminergic probe. Archives of General Psychiatry, 62(11), 1228-1236.

Turkeltaub, P. E., Eden, G. F., Jones, K. M., \& Zeffiro, T. A. (2002). Meta-analysis of the functional neuroanatomy of single-word reading: method and validation. NeuroImage, 16(3 Pt 1), 765-780.

Turkeltaub, P. E., Eickhoff, S. B., Laird, A. R., Fox, M., Wiener, M., \& Fox, P. (2012). Minimizing within-experiment and within-group effects in activation likelihood estimation meta-analyses. Human Brain Mapping, 33(1), 1-13.

Ubl, B., Kuehner, C., Kirsch, P., Ruttorf, M., Diener, C., Flor, H. (2015). Altered neural reward and loss processing and prediction error signalling in depression. Soc Cogn Affect Neurosci.

Ursu, S., Kring, A. M., Gard, M. G., Minzenberg, M. J., Yoon, J. H., Ragland, J. D., et al. (2011). Prefrontal cortical deficits and impaired cognition-emotion interactions in schizophrenia. The American Journal of Psychiatry, 168(3), 276-285.

van den Bos, W., Rodriguez, C. A., Schweitzer, J. B., \& McClure, S. M. (2014). Connectivity strength of dissociable striatal tracts predict individual differences in temporal discounting. Journal of Neuroscience, 34(31), 10298-10310.

Vijayaraghavan, L., Vaidya, J. G., Humphreys, C. T., Beglinger, L. J., \& Paradiso, S. (2008). Emotional and motivational changes after bilateral lesions of the globus pallidus. Neuropsychology, 22(3), 412418.

Wager, T. D., Lindquist, M., \& Kaplan, L. (2007). Meta-analysis of functional neuroimaging data: current and future directions. Social Cognitive and Affective Neuroscience, 2(2), 150-158.

Wallis, J. D., \& Kennerley, S. W. (2010). Heterogeneous reward signals in prefrontal cortex. Current Opinion in Neurobiology, 20(2), 191198.

Walter, H., Kammerer, H., Frasch, K., Spitzer, M., \& Abler, B. (2009a). Altered reward functions in patients on atypical antipsychotic medication in line with the revised dopamine hypothesis of schizophrenia. Psychopharmacology, 206(1), 121-132.

Walter, M., Henning, A., Grimm, S., Schulte, R. F., Beck, J., Dydak, U., et al. (2009b). The relationship between aberrant neuronal activation in the pregenual anterior cingulate, altered glutamatergic metabolism, and anhedonia in major depression. Archives of General Psychiatry, 66(5), 478-486. 
Waltz, J. A., Schweitzer, J. B., Gold, J. M., Kurup, P. K., Ross, T. J., Salmeron, B. J., et al. (2009). Patients with schizophrenia have a reduced neural response to both unpredictable and predictable primary reinforcers. Neuropsychopharmacology, 34(6), 1567-1577.

Waltz, J. A., Schweitzer, J. B., Ross, T. J., Kurup, P. K., Salmeron, B. J., Rose, E. J., et al. (2010). Abnormal responses to monetary outcomes in cortex, but not in the basal ganglia, in schizophrenia. Neuropsychopharmacology, 35(12), 2427-2439.

Wang, X., Yao, S., Kirkpatrick, B., Shi, C., \& Yi, J. (2008). Psychopathology and neuropsychological impairments in deficit and nondeficit schizophrenia of chinese origin. Psychiatry Research, 158(2), 195-205.

Whitton, A. E., Treadway, M. T., \& Pizzagalli, D. A. (2015). Reward processing dysfunction in major depression, bipolar disorder and schizophrenia. Current Opinion in Psychiatry, 28(1), 7-12.
Yang, X. H., Huang, J., Zhu, C. Y., Wang, Y. F., Cheung, E. F. C., Chan, R. C. K., et al. (2014). Motivational deficits in effort-based decision making in individuals with subsyndromal depression, first-episode and remitted depression patients. Psychiatry Research, 220(3), 874882.

Zhang, W. N., Chang, S. H., Guo, L. Y., Zhang, K. L., \& Wang, J. (2013). The neural correlates of reward-related processing in major depressive disorder: a meta-analysis of functional magnetic resonance imaging studies. Journal of Affective Disorders, 151(2), 531-539.

Zhu, X., Wang, X., Xiao, J., Liao, J., Zhong, M., Wang, W., et al. (2012). Evidence of a dissociation pattern in resting-state default mode network connectivity in first-episode, treatment-naive major depression patients. Biological Psychiatry, 71(7), 611-617. 